\title{
Difference Metabolites Induced By Deoxynivalenol in Serum and Urine of Weaned Rabbits Detected Using LC-MS Based Metabolomics
}

Fuchang Li

Shandong Agricultural University

Libo Huang

Shandong Agricultural University

Quancheng Liu

Shandong Agricultural University

Pengwei Wang

Shandong Agricultural University

Hongju Chen

Shandong Agricultural University

Chunyang Wang ( $\nabla$ wcy@sdau.edu.cn )

Shandong Agricultural University https://orcid.org/0000-0002-6707-2274

Research

Keywords: Deoxynivalenol, weaned rabbits, metabolomics, pathogenic mechanism, biomarker

Posted Date: November 18th, 2020

DOI: https://doi.org/10.21203/rs.3.rs-107751/v1

License: (9) (i) This work is licensed under a Creative Commons Attribution 4.0 International License.

Read Full License 
1 Difference metabolites induced by Deoxynivalenol in

2 serum and urine of weaned rabbits detected using

3 LC-MS based metabolomics

4

$5 \quad$ Fuchang $\mathrm{Li}^{1, \#}$, Libo Huang ${ }^{1, \#}$, Quancheng Liu ${ }^{1,}$ Pengwei Wang ${ }^{1}$, Hongju Chen $^{1}$,

6 Chunyang Wang ${ }^{1, *}$

7

8

9

10

11

12

13

14

15

16

17

18

19

20

21

22

23

24

25

26

27

28

29

30

31

32

33

34

35

36

37

38

${ }^{1}$ Shandong Provincial Key Laboratory of Animal Biotechnology and Disease Control and Prevention,

College of Animal Science and Veterinary Medicine, Shandong Agricultural University, Taian, Shandong, 271018, China. " Co-first author: Fuchang Li and Libo Huang contributed equally to this work.

* Correspondence: +86-13-50548-0263; wcy@sdau.edu.cn

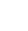

(1)
6 7

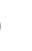
0

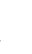
3 34 7 38 


\section{Abstract}

Background: The main toxin effects of Deoxynivalenol (DON), which known as one of the mycotoxins with the highest pollution rate, is the result of long-term accumulation, and there are no obvious clinical signs at the early stage. Specific metabolites in blood and urine can be used as biomarkers and become an important diagnostic indicator for DON poisoning monitoring. At present, studies on the metabolic pathways and characteristics of DON mainly focus on humans, pigs and poultry, but few study on rabbits. This study aims to reveal the difference in DON-induced metabolites in the serum and urine of weaned rabbits, so as to help find potential biomarkers and understand the mechanism of DON in rabbits.

Methods: A total of 32 weaned rex rabbits were divided evenly into two groups, namely the control group and DON group. Both groups of rabbits were fed with the basic diet. Rabbits in DON group were intraperitoneally injected with DON at $1.5 \mathrm{mg} / \mathrm{kg}$ b.w. every two days before feeding, while rabbits in control group were injected with saline at 1.5 $\mathrm{mg} / \mathrm{kg}$ b.w. in the same way. After the 25-day trial, the serum and urine samples at different experimental period were collected for LC/MS analysis.

Results: The results based on the LC-MS/MS method showed that DON can be metabolized rapidly in blood, and urine is the main metabolism pathway for DON. The data based on metabolomics illustrated that underlying biomarkers in serum were mainly involved in Glycerophospholipid metabolism, Tryptophan metabolism and Pentose and glucuronate interconversions, while those in urine samples involved in Caffeine metabolism, Glycine, serine and threonine metabolism, and Terpenoid backbone 
61 biosynthesis. Correlation analysis suggested that DON can induce the changes in certain 62 disease-related metabolites in serum and urine.

63 Conclusions: The pathogenic mechanism of DON includes multiple levels, indicating 64 that DON poisoning is caused by multiple factors acting on multiple links.

65 Keywords: Deoxynivalenol, weaned rabbits, metabolomics, pathogenic mechanism, biomarker. 


\section{Background}

Contamination of agricultural products caused by natural mycotoxins has been a long-standing challenge for agriculture and food industries [1]. Deoxynivalenol (DON), widely detected in cereal grains and animal feed worldwide, is considered to be a underlying health hazard for humans and animals [2,3]. Consuming contaminated DON can induce physiological abnormalities, including digestive disorders, intestinal-barrier damage, immune disruption and reproductive toxicity, etc $[4,5]$.

Toxicokinetics studies have shown that DON ingested orally can be rapidly absorbed into the blood circulation through the body's small intestinal barrier, then distribute to peripheral organs, as well as affect the function of histocyte [6]. DON is degraded into a variety of metabolic products in the digestive tract by microorganisms or in the intestinal mucosa, liver, kidney and other organs of the body [7]. The metabolic characteristics of DON in humans and animals, including the toxicological kinetics of DON absorption, distribution, metabolism and excretion, are the basis for the toxicity assessment, toxicity mechanism and intervention techniques of DON [8]. Therefore, studies concerning the metabolic characteristics of DON have attracted wide attention. Compared with T2 toxin of the congeneric trichothecenes, the identified metabolites of DON are relatively less, mainly including: DON-GlcA, DON-sulfonate and DON-sulfate etc $[9,10]$. Among them, de-epoxidation deoxynivalenol (DOM), mainly acted as a type of metabolite produced by DON under the catalysis of intestinal microorganisms, which is most common metabolite of DON in different species (such as humans, rodents, pigs, chickens and ruminants) $[11,12]$. Therefore, it is generally believed that DOM-1 is the most typical biomarker of 
DON and has been applied in relevant studies, such as the evaluation of metabolism and biological detoxification of DON and so on $[13,14]$. However, detection cost of DOM-1 is relatively high, and further research is needed to reveal whether there are other biomarker in DON.

Clinically, most DON poisoning is the result of long-term accumulation, and there are no obvious clinical signs at the early stage [15]. Therefore, it is particularly urgent to find new specific and highly sensitive diagnostic indicators and methods. Blood and urine are the most common physiological samples in clinical tests. The distribution and elimination rate of DON and its metabolites in blood and urine is important parameters for its pathogenicity[8]. Specific metabolites in blood and urine can be used as biomarkers and become an important diagnostic indicator for DON poisoning monitoring [16,17]. Metabolic characteristics of DON in animals and description of metabolites related to pathological conditions may be of great significance for understanding mechanisms and pathways of DON, discovering biomarkers and establishing early diagnosis and intervention [10,18]. Metabonomics is based on the modern molecular biology technology and the method of combining the computer information technology, all metabolic components in the cell, tissue or organ, especially for small molecular substances monitoring, analyzes the source of the metabolites path, and make the metabolism network topology, is currently the disease diagnosis, drug development and toxicology analysis such as one of the most promising technology $[19,20]$. However, there are few studies on the mechanism of action of mycotoxins such as DON based on metabonomics. 

and it is also a kind of high quality source of meat. With the continuous expansion of rabbit breeding scale and the wide application of full price feed in rabbit industry, the issue concerning contamination of mycotoxins in feed and potential threats to food safety have attracted more and more attention from scientists [21]. As a kind of monogastric herbivores and hindgut fermenter animal [22,23], the effect of the special intestinal structure of rabbits on DON metabolism is not clear. At present, studies on the toxic effects of DON mainly focus on humans, pigs and poultry, but studies on the metabolic pathways and characteristics of DON in rabbits are very limited. Therefore, in this study, the blood and urine samples of DON poisoning weaned rabbits will be analyzed using the research strategy of LC-MS metabolomics, and the endogenous metabolites between understand the mechanism of DON action in rabbits.

Methods

\section{Animal Experiment and Sample Collection}

Ingredient composition of the feedstuff of weaned rabbits supplied by Fat Rabbits Feed Company (Taian, Shandong), as well as the concentration of DON, Zearalenone (ZEA) and Aflatoxin B1 (AFB1), is listed in Table 1. DON standards were diluted to $1.5 \mathrm{mg} / \mathrm{mL}$ using sterile ultrapure water for later use. A total of 32 weaned rex rabbits (35-day-old, male and female half) were divided evenly into two groups based on their weight, namely the control group and DON group. Both groups of experimental rabbits, 
individually placed in metabolic cages, were fed with the basic diet. Weaned rabbits in the DON group were intraperitoneally injected with $1.5 \mathrm{mg} / \mathrm{kg}$ b.w. DON every two days before feeding in the morning, while the rabbits in the control group were injected with the same amount of normal saline every two days in the same way. The experiment lasted for 25 days, that is a total of 12 injections to rabbits by the end of the trail. On day 8, day 16 and 24 of experiment, 16 rabbits (8 rabbits each group) were selected for blood collection by ear vein before injecting DON (0h), as well as $1 \mathrm{~h}, 2 \mathrm{~h}, 3 \mathrm{~h}, 4 \mathrm{~h}$ and $6 \mathrm{~h}$ after injecting DON, respectively. All collected blood coagulated naturally and centrifuged at $1500 \mathrm{~g}$ for $10 \mathrm{~min}$ in order to acquire serum samples, which stored at $-70{ }^{\circ} \mathrm{C}$ for further analysis. On day 8, day 16 and 24 of experiment, the urine samples of 24 rabbits at empty stomach were collected, which centrifuged at 2,500 $\mathrm{g}$ for $10 \mathrm{~min}$ and stored at $-70{ }^{\circ} \mathrm{C}$ until sample procession, while the feces samples were collected and stored at $-70{ }^{\circ} \mathrm{C}$ after drying at the same day. On day 25 of experiment, the serum and urine samples without injecting DON were collected for LC/MS analysis.

Table 1 The ingredient composition of the basic diets

\begin{tabular}{llll}
\hline \multicolumn{1}{c}{ Ingredient (\%) } & \multicolumn{3}{c}{ Calculated composition } \\
\hline Maize & 14 & Dry matter & 88.64 \\
Soybean meal & 17 & Crude protein & 20.05 \\
Wheat bran & 13 & Crude fibre & 18.78 \\
Corn germ meal & 19 & Crude ash & 10.45 \\
Rice hulls & 10 & Crude fat & 3.34 \\
Soybean straw powder & 7 & Calcium & 0.72 \\
alfalfa & 10 & Total Phosphorus & 0.55 \\
Malt Sprout & 5 & Digestible energy ( MJ/kg) & 10.06 \\
Sweet wormwood & 3.5 & & \\
Premix material ${ }^{1}$ & 1.5 & & \\
Total & 100 & & \\
\hline \multicolumn{5}{c}{ Content of $\mathbf{M y c o t o x i n ~} \quad(\boldsymbol{\mu g} / \mathbf{k g})^{\mathbf{2}}$} \\
\hline Deoxynivalenol (DON) & 23.18 & \\
Zearalenone (ZEA) & 135.26 & \\
Aflatoxin B1 ( AFB1) & 7.08 & & \\
\hline
\end{tabular}




\section{Liquid chromatography-tandem mass spectrometry (LC-MS/MS)}

Standards of DON $\left(\mathrm{C}_{15} \mathrm{H}_{20} \mathrm{O}_{6}\right)$ and DOM-1 $\left(\mathrm{C}_{15} \mathrm{H}_{20} \mathrm{O}_{5}\right)$ purchased from Triplebond Company (Guelph, Guelph, Canada). Sample preparation steps referred to the method described by Brezina et al. (2014) [24]. Contents of DON and DOM-1 were detected via LC-MS/MS following the protocol reported by Li et al. (2017) [14]. These detection procedures were performed in the Institute of Quality Standards and Detection Technology, Chinese Academy of Agricultural Sciences.

\section{Preparation of serum and urine samples}

All chemicals regeants used in the present study were analytically pure or chromatographic grade. Methanol, chloroform, formic acid, water, acetonitrile were purchased from CNW Company in German. 1-2-chlorophenylalanine was purchased from Hengchuang Biotechnology Company (Shanghai, China). In this study, 16 serum and 16 urine samples from the two test groups were used for metabolomics analysis. Firstly, $10 \mu \mathrm{L}$ of 2-chloro-l-phenylalanine $(0.3 \mathrm{mg} / \mathrm{mL})$ was dissolved in methanol as internal standard, then mixed accurately $100 \mu \mathrm{L}$ serum samples and vortexed for $10 \mathrm{~s}$. Secondly, after $300 \mu \mathrm{L}$ of pre-cooling mixed solution with methanol and acetonitrile (2:1, $\mathrm{v} / \mathrm{v}$ ) was added and vortexed for $1 \mathrm{~min}$, these mixtures were extracted using ultrasound in ice water bath for $10 \mathrm{~min}$. Thirdly, these mixed solution was centrifuged at 13,000 rpm for $15 \mathrm{~min}$ after stood at $-20{ }^{\circ} \mathrm{C}$ for $30 \mathrm{~min}$, then $200 \mu \mathrm{L}$ extracted supernatant were filtered with $0.22 \mu \mathrm{m}$ pinhole filters and transferred to LC vial, which were stored at $-80^{\circ} \mathrm{C}$ for future step.Lastly, all samples were mixed to be pooled sample at a certain ratio, which acted as quality control samples (QC) sample and used to verify the results in 
present research. For urine samples, the treatment procedure was in keeping with the serum proceeding, except that $150 \mu \mathrm{L}$ urine specimen was centrifuged at $13,000 \mathrm{rpm}$ for 10 min firstly, and then $100 \mu \mathrm{L}$ supernatant was accurately extracted and added into the mix solution with internal standard.

\section{LC-MS analysis}

Metabolic profiling was monitored through an ACQUITY UPLC system (Waters Corporation, Milford, USA) coupled with a Triple TOF 5600 System (AB SCIEX, Framingham, MA). Meanwhile, ACQUITY UPLC BEH C8 (100 mm ×2.1mm, 1.7 um) was applied in ESI positive ion mode, while ACQUITY UPLC HSS T3 $(100 \mathrm{~mm} \times 2.1 \mathrm{~mm}$, 1.8um ) was used in negative ion mode. For positive ion mode, Mobile phase A was water including $0.1 \%$ formic acid and B was acetonitrile with $0.1 \%$ formic acid, while for negative ion mode, mobile phase A was water including $6.5 \mathrm{mM}$ ammonium bicarbonate and $\mathrm{B}$ was water and 95\% methanol containing $6.5 \mathrm{mM}$ ammonium bicarbonate. The temperature of the column was $50^{\circ} \mathrm{C}$, the injection volume was $5 \mu \mathrm{L}$ and flow rate was at $0.35 \mathrm{~mL} / \mathrm{min}$. The elution gradient of HPLC and parameters of mass spectrum in this experiment were listed in Table S1 and Table S2, respectively. Throughout the whole analysis process, the QC samples were inserted at every 8 samples so as to evaluate the repeatability.

\section{Data processing}

The raw data were transformed to mzML format via MSconventer, then peak extraction was carried out by XCMS software. All data from positive and negative ion modes were 
combined into a data matrix, which contains all the information extracted from the raw data that can be used for subsequent analysis. The data matrix was exported into the SIMCA software (Version 14.0, Umetrics, Sweden), then unsupervised principal component analysis (PCA) was performed to observe the samples between the population distribution and stability of the whole process of analysis, while supervised orthogonal partial least-squares discriminant analysis (OPLS-DA) was used to distinguish the overall difference in metabolic profile and screen the difference metabolites between groups. For OPLS-DA analysis, variable important in projection (VIP) greater than 1 were regarded as significant variables. The differential metabolites between groups were screened on the basis of the combination of multidimensional analysis and one-dimensional analysis. The screening criterion was the VIP values larger than 1 , coupling with $\mathrm{p}$ value from student's $\mathrm{t}$ test less than 0.05 . In addition, the fold change (FC) values referred to the ratio of the average content of metabolites in two groups. Differential metabolites were identified by the rapid identification and analysis software system (OSI/SMMS, Dalian, China). Analysis of identified metabolites and pathway enrichment was based on public databases, including HMDB (http://www.hmdb.ca), KEGG (http://www.genome.jp/kegg/) and LipidMaps (http://www.lipidmaps.org/) databases.

\section{Statistical analysis}

The test data were analyzed by one-way ANOVA and $t$ test, which expressed using mean \pm standard deviation (SD). $p<0.05$ was considered statistically significant, and $p<0.01$ was considered extremely significant. 


\section{Results and disscusion}

DON concentration in rabbit serum samples of different experimental periods.

Studies have shown that the absorptivity of DON varied greatly among animals, for example, the absorptivity of pigs, chickens, sheep and cattle was $82 \%, 19 \%, 9.9 \%$ and $1 \%$, respectively $[10,25]$. According to statistics, DON can reach the peak in blood after 15-30 min oral intake in pig, and reach the absorption peak within 3-4 $\mathrm{h} \mathrm{[26].} \mathrm{The} \mathrm{results} \mathrm{of} \mathrm{this}$ study showed that the changes of DON concentration in serum of rabbits on day 8,16 and 24 exhibited a highly consistent trend after DON was injected (Fig.1). Namely, the content of DON in serum before injection was consistent with that of the control group, while the content of DON in serum increased rapidly after injection, reached the peak at $1 \mathrm{~h}$ after injection, and then decreased rapidly. At 4 hours after injection, the concentration of DON was close to the control group, and 6 hours after injection, the concentration was almost the same as that of the control group. Moreover, with the increase of injection days, the concentration of DON did not change significantly in the same period, indicating that DON can be rapidly metabolized in the blood, with almost no cumulative effect. This result suggested that, in the clinical monitoring of DON poisoning, the collection of blood samples has a strong timeliness, that is, blood indicators within $1-2 \mathrm{~h}$ after ingestion are more meaningful for detection.

\section{Concentration of DON in ruine and feces samples}

In this study, the content of DON in urine samples (Fig.2A) was 1,205.9 ng/ml, 1,590.65 $\mathrm{ng} / \mathrm{ml}$ and $1,797.78 \mathrm{ng} / \mathrm{ml}$ respectively on the 8 th, 16 th and 24 th day after DON administration, while that in feces (Fig.2B) was $438 \mathrm{ng} / \mathrm{ml}, 1,213.4 \mathrm{ng} / \mathrm{ml}$ and 
$3,457.38 \mathrm{ng} / \mathrm{ml}$, respectively. In addition, with the increase of the number of DON injections, the concentration of DON in urine and feces showed a significant trend of increase, especially in feces. Interestingly, on the 8th day of the experiment, the concentration of DON in feces was significantly lower than that in urine samples during the same period, but the trend of increase was obvious. On the 24th day, the concentration of DON in feces was significantly higher than that in urine, indicating that with the prolongation of DON exposure period, the intestine becomes the main organ for DON storage and excretion.

\section{Concentration of DOM-1 in ruine and feces samples}

Microorganisms in the digestive tracts of animals and humans can degrade the toxic DON to the non-toxic DOM-1, which usually as the most typical biomarker of DON $[27,28]$. On the 8 th, 16 th and 24 th day of the experiment, the content of DOM-1 in the urine of DON group was $108.03 \mathrm{ng} / \mathrm{ml}, 129.75 \mathrm{ng} / \mathrm{ml}, 135.4 \mathrm{ng} / \mathrm{ml}$ (Fig.2C), and the content of DOM-1 in feces was $57.13 \mathrm{ng} / \mathrm{ml}, 78.8 \mathrm{ng} / \mathrm{ml}, 130.3 \mathrm{ng} / \mathrm{ml}$ (Fig.2D), respectively, which were significantly higher than those in the control group in the same period. Moreover, with the increase of the number of DON injections, the concentration of DOM-1 in urine and feces showed a significant trend of increase, and the content of DOM-1 in urine was higher than that in feces in the same period. The amount of DOM-1 excreted in urine and feces were only about $5 \%-10 \%$ of the amount of DON excreted in the same period, indicating that a large amount of DON were still excreted in the form of DON after entering the body, and only a small amount will be decomposed into the secondary metabolite DOM-1. The distribution of bacterial flora in the small intestine of 
pigs are relatively less, as well as the degradation rate from DON to DOM-1 is also relatively lower compared with poultry or ruminants, thus pigs are more sensitive to DON [29]. Previous experiments proved that the destruction induced by DON in the anterior segment of the small intestine for rabbits more seriouser than to the posterior segment, suggesting that part of DON in the posterior segment is converted to DOM-1 by intestinal microflora, thus relieve the destruction to the ileum [14].

\section{Multivariate analysis of LC-MS data}

Compared with the traditional HPLC-MS method, the LC-MS adopted in this study has higher peak volume, separation degree and sensitivity, so it is more suitable for the analysis of metabonomics for serum and urine samples of weaned rabbits. Test data of serum and urine were uploaded severally into SIMCA for multivariate analysis in present research. PCA was applied to analyze the entire allocation between samples and stability of the whole analytic processing [18]. PCA score plots of total serum and urine between two groups illustrated that the clustering of endogenous metabolites in the DON group significantly changed compared with the control group (Fig.3A and Fig.3B). In addition, it can be seen from Table 2 that both $R^{2} X$ of serum $\left(R^{2} X=0.543\right)$ and urine $\left(R^{2} X=0.662\right)$ were greater than 0.5 , indicating that the model was reliable. OPLS-DA of and Fig.3D), either serum or urine samples between two groups were completely separated, further indicating the metabolic characteristics in serum and urine were serum and urine samples were established respectively to further validate separation of the metabolic profiles between two groups. As showed in OPLS-DA score plots (Fig.3C significantly affected by DON addition. Furthermore, data from OPLS-DA model (Table 

greater than 0.5, indicating that the OPLS-DA model was well established. Meanwhile, the results from 200 response permutation testing (RPT), which were used to examine the quality of the model in order to prevent the model from overfitting, indicated that the OPLS-DA models were well established (Fig.3E and Fig.3F). In brief, the multivariate analysis of LC-MS data proved that the data quality was reliable and the models were well established, as well as there were a significant difference between two groups, indicating the metabolic characteristics in serum and urine were significantly affected by

Table 2 Model quality parameters of multivariate analysis in serum and urine samples between two groups

\begin{tabular}{ccccc}
\hline Type & $\mathrm{N}$ & $\mathrm{R}^{2} X(\mathrm{cum})$ & $\mathrm{R}^{2} \mathrm{Y}(\mathrm{cum})$ & $\mathrm{Q}^{2}$ (cum) \\
\hline Serum & & & & \\
PCA & 16 & 0.543 & & 0.183 \\
OPLS-DA & 16 & 0.577 & 0.965 & 0.768 \\
Urine & & & & \\
PCA & 16 & 0.662 & & 0.37 \\
OPLS-DA & 16 & 0.527 & 0.993 & 0.956 \\
\hline
\end{tabular}

Notes: $N$ means the number of samples analyzed. $R^{2} X$ and $R^{2} Y$ refers to the cumulative interpretation rate of the model in the $\mathrm{X}$-axis and $\mathrm{Y}$-axis when modeling with the multivariate statistical analysis, or can be understood as the square of the percentage of the original data information retained at the $\mathrm{X}$-axis or $\mathrm{Y}$-axis direction, respectively. $\mathrm{Q}^{2}$ means the parameters of the response ranking test, applying for measure whether the model is over-fitting.

\section{Screening of differential metabolites}

291 The differential metabolites between groups were screened on the basis of the combination of multi-dimensional analysis and single-dimensional analysis. According to VIP $\geqslant 1$ coupled with $p<0.05$ as the screening criteria in this study, total of 179 serum 
view of FC $>1$ means up-regulation and FC $<1$ means down-regulation of metabolites, 51 metabolites were upregulated and 128 were up-regulated in serum, while 327 metabolites were upregulated and 199 were down-regulated in urine samples. As a result, 26 metabolites in serum, as well as 36 metabolites in urine respectively were screened as potential biomarkers based on the principle of $\mathrm{p}$ values from small to large (Table 3 and Table 4). Furthermore, all the differential metabolites in serum and urine of rabbits were analyzed applying VENN method, and the results showed that there are 15 differential metabolites shared by serum and urine (Fig.4). As displayed in Table 5, these 15 shared metabolites showed different regulatory trends in serum and urine. Although both urine and blood are considered as the important physiological samples, the metabolic pathways of DON in serum and urine were quite cxdedifferent in our research, and the difference in urine was greater than that in blood obviously. Additionally, detection of DON in urine was less dependent on the time of feeding, so we suggest that the relevant detective indicators in urine may be more meaningful for clinical monitoring of DON.

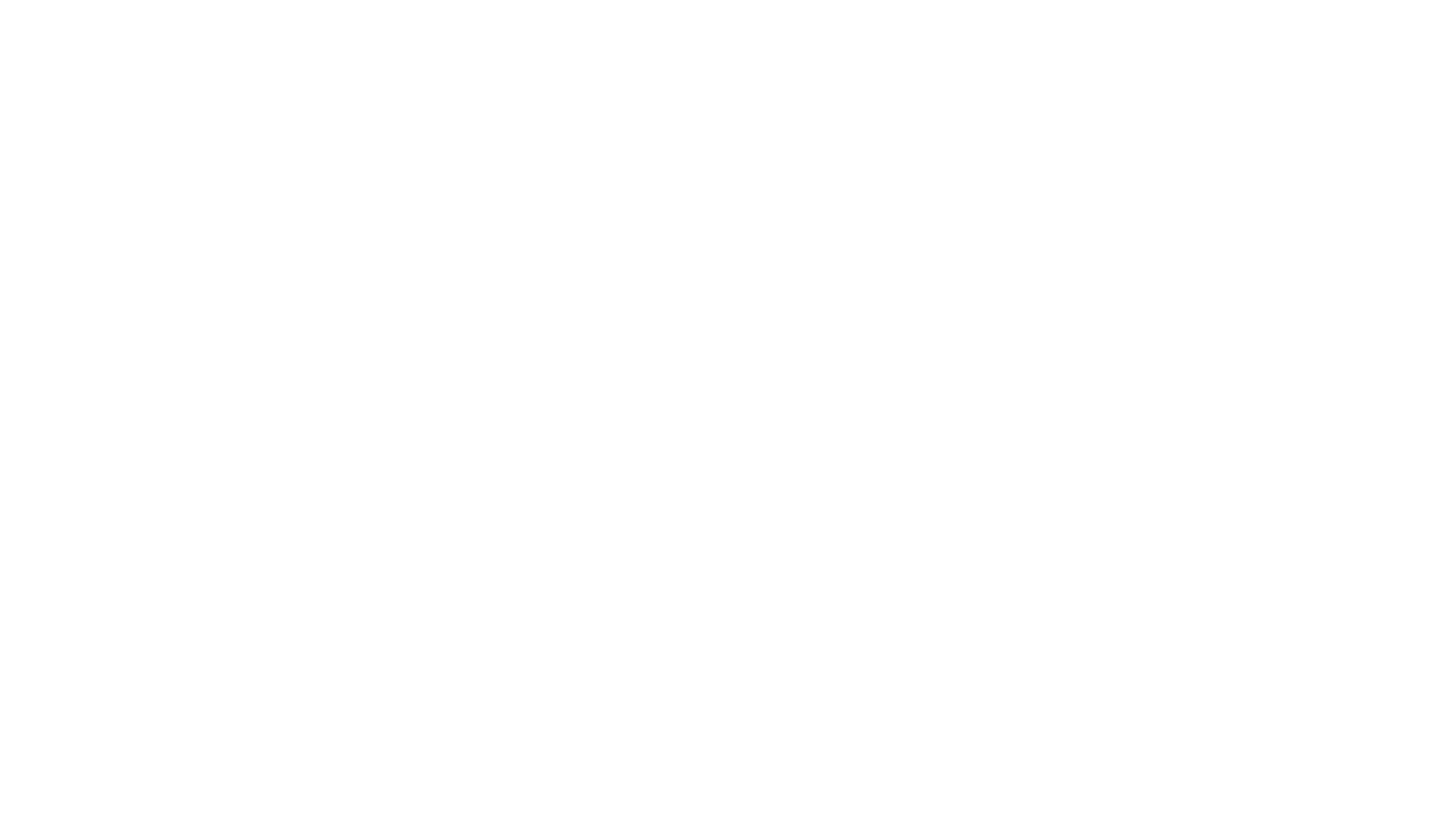




\begin{tabular}{|c|c|c|c|c|c|}
\hline NO. & $m / z$ & Metabolites & VIP & FC & Trend \\
\hline 1 & 225.18 & Xylometazoline & 1.038 & 2.159 & $\uparrow * *$ \\
\hline 2 & 251.20 & oxohexadecanoic acid & 1.139 & 2.079 & $\uparrow * *$ \\
\hline 3 & 253.22 & Cadinene & 4.309 & 2.077 & $\uparrow * *$ \\
\hline 4 & 267.23 & Avocadene & 1.612 & 1.603 & $\uparrow * *$ \\
\hline 5 & 476.11 & Ochratoxin c & 1.434 & 1.539 & $\uparrow * *$ \\
\hline 6 & 511.47 & 10-hydroxy-16-hentriacontanone & 2.105 & 1.527 & $\uparrow^{* *}$ \\
\hline 7 & 541.26 & Cortolone-3-glucuronide & 1.168 & 3.291 & $\uparrow * *$ \\
\hline 8 & 639.54 & Pregnanediol & 3.106 & 2.260 & $\uparrow^{* *}$ \\
\hline 9 & 763.50 & Narasin & 1.262 & 1.661 & $\uparrow^{* *}$ \\
\hline 10 & 787.60 & Tetradecanoylcarnitine & 1.339 & 1.734 & $\uparrow^{* *}$ \\
\hline 11 & 851.52 & Prostaglandin pge2 1 -glyceryl ester & 1.795 & 2.059 & $\uparrow^{* *}$ \\
\hline 12 & 862.61 & 3-o-sulfogalactosylceramide & 7.265 & 2.947 & $\uparrow * *$ \\
\hline 13 & 863.57 & Latanoprost & 7.242 & 3.996 & $\uparrow^{* *}$ \\
\hline 14 & 153.02 & 2,4-dihydroxybenzoic acid & 1.926 & 0.307 & $\downarrow * *$ \\
\hline 15 & 178.05 & Hippuric acid & 2.254 & 0.420 & $\downarrow * *$ \\
\hline 16 & 359.19 & Tricyclodehydroisohumulone & 2.196 & 0.475 & $\downarrow * *$ \\
\hline 17 & 417.12 & Phlorizin & 1.630 & 0.313 & $\downarrow * *$ \\
\hline 18 & 457.33 & N-decanoylglycine & 2.642 & 0.414 & $\downarrow * *$ \\
\hline 19 & 464.30 & Glycocholic acid & 2.215 & 0.300 & $\downarrow * *$ \\
\hline 20 & 465.30 & Cholesterol sulfate & 3.711 & 0.658 & $\downarrow * *$ \\
\hline 21 & 466.31 & Gamma-linolenyl carnitine & 2.558 & 0.626 & $\downarrow * *$ \\
\hline 22 & 499.30 & Corosin & 1.670 & 0.599 & $\downarrow * *$ \\
\hline 23 & 528.26 & glycine & 2.455 & 0.201 & $\downarrow * *$ \\
\hline 24 & 772.62 & Arachidonoyl serinol & 5.511 & 0.581 & $\downarrow * *$ \\
\hline 25 & 776.58 & Leukotriene b4 ethanolamide & 2.946 & 0.612 & $\downarrow * *$ \\
\hline 26 & 833.54 & 11-hydroxyeicosatetraenoate glyceryl ester & 10.444 & 0.204 & $\downarrow * *$ \\
\hline
\end{tabular}

Notes: VIP means variable importance in projection. FC refers to the fold change, and FC $>1$ or $\mathrm{FC}<1$, indicated by up arrows or down arrows repectively, were usd to represent latively increased or decreased levels of the metabolites. ${ }^{* *}$ means $\mathrm{p}<0.01$ between groups. 


\begin{tabular}{|c|c|c|c|c|c|}
\hline NO. & $m / z$ & Metabolites & VIP & FC & Trend \\
\hline 1 & 146.060 & 4-Hydroxyquinoline & 3.615 & 4.440 & $\uparrow * *$ \\
\hline 2 & 153.019 & dihydroxybenzoic acid & 4.667 & 2.972 & $\uparrow * *$ \\
\hline 3 & 181.992 & Saccharin & 5.979 & 5.954 & $\uparrow * *$ \\
\hline 4 & 201.023 & Methylfuran & 5.976 & 2.467 & $\uparrow * *$ \\
\hline 5 & 279.134 & Asp Ala Gly & 7.454 & 3.436 & $\uparrow * *$ \\
\hline 6 & 281.120 & Asp-phe & 3.989 & 2.372 & $\uparrow * *$ \\
\hline 7 & 325.128 & Dihydroconiferin & 10.869 & 5.370 & $\uparrow * *$ \\
\hline 8 & 327.142 & S-methylmethionine & 5.209 & 4.400 & $\uparrow * *$ \\
\hline 9 & 339.093 & 3'-ketolactose & 3.560 & 4.238 & $\uparrow * *$ \\
\hline 10 & 344.172 & His Gly Asn & 4.867 & 5.016 & $\uparrow * *$ \\
\hline 11 & 385.186 & Pisumionoside & 3.835 & 3.193 & $\uparrow * *$ \\
\hline 12 & 401.164 & Clusin & 4.624 & 2.818 & $\uparrow * *$ \\
\hline 13 & 411.199 & Adapalene & 4.274 & 2.887 & $\uparrow * *$ \\
\hline 14 & 413.214 & Miglitol & 5.521 & 3.377 & $\uparrow * *$ \\
\hline 15 & 413.215 & Buclizine & 4.259 & 2.579 & $\uparrow * *$ \\
\hline 16 & 413.215 & His Glu Lys & 3.478 & 2.696 & $\uparrow * *$ \\
\hline 17 & 445.077 & 2'-Hydroxyisoorientin & 6.132 & 4.572 & $\uparrow * *$ \\
\hline 18 & 447.094 & Baicalin & 7.575 & 4.450 & $\uparrow * *$ \\
\hline 19 & 187.007 & P-cresol sulfate & 4.568 & 0.427 & $\downarrow * *$ \\
\hline 20 & 194.081 & N-Phenylacetylglycine & 5.480 & 0.520 & $\downarrow * *$ \\
\hline 21 & 212.005 & Indoxyl sulfate & 9.501 & 0.509 & $\downarrow * *$ \\
\hline 22 & 255.232 & Ethyl tetradecanoate & 8.553 & 0.519 & $\downarrow * *$ \\
\hline 23 & 281.119 & Phenprocoumon & 3.557 & 0.573 & $\downarrow * *$ \\
\hline 24 & 285.113 & Matricarin & 9.843 & 0.418 & $\downarrow * *$ \\
\hline 25 & 295.097 & Gamma-l-glutamyl-1-methionine sulfoxide & 6.878 & 0.374 & $\downarrow * *$ \\
\hline 26 & 301.143 & 6-ketoestriol & 3.694 & 0.210 & $\downarrow * *$ \\
\hline 27 & 313.107 & Sappanone A Dimethyl ether & 7.724 & 0.353 & $\downarrow * *$ \\
\hline 28 & 313.274 & Ricinoleic Acid methyl ester & 4.475 & 0.570 & $\downarrow * *$ \\
\hline 29 & 315.123 & Sorgolactone & 8.085 & 0.567 & $\downarrow * *$ \\
\hline 30 & 329.102 & 2'-Hydroxy-4',6'-dimethoxychalcone & 12.136 & 0.426 & $\downarrow * *$ \\
\hline 31 & 331.284 & Glycerol 1-hexadecanoate & 3.942 & 0.575 & $\downarrow * *$ \\
\hline 32 & 338.342 & Erucamide & 6.969 & 0.562 & $\downarrow * *$ \\
\hline 33 & 341.305 & Hexadecyl Acetyl Glycerol & 3.293 & 0.577 & $\downarrow * *$ \\
\hline 34 & 348.144 & Val Asp Asp & 8.709 & 0.453 & $\downarrow * *$ \\
\hline 35 & 349.148 & Trp Ser Gly & 5.076 & 0.422 & $\downarrow * *$ \\
\hline 36 & 353.100 & Americanola & 5.193 & 0.345 & $\downarrow * *$ \\
\hline
\end{tabular}

Notes: VIP means variable importance in projection. FC refers to the fold change, and FC $>1$ or FC $<1$, indicated by up arrows or down arrows repectively, were usd to represent latively increased or decreased levels of the metabolites. ${ }^{* *}$ means $\mathrm{p}<0.01$ between groups. 


\begin{tabular}{lccccccc}
\hline \multirow{2}{*}{\multicolumn{1}{c}{ Shared metabolites }} & \multicolumn{2}{c}{ VIP } & \multicolumn{2}{c}{ FC } & \multicolumn{2}{c}{ Trend } \\
\cline { 2 - 7 } & Serum & Urine & Serum & Urine Serum & Urine \\
\hline Dl-Indole-3-lactic acid & 1.10 & 1.62 & 1.89 & 0.73 & $\uparrow * *$ & $\downarrow * *$ \\
N-[(3a,5b,7a)-3-hydroxy-24-oxo-7-(sulfooxy) & & & & & & \\
cholan-24-yl]-glycine & 4.88 & 2.45 & 0.35 & 0.20 & $\downarrow * *$ & $\downarrow * *$ \\
2'-Hydroxy-4',6'-dimethoxychalcone & 12.14 & 2.02 & 0.43 & 0.47 & $\downarrow * *$ & $\downarrow * *$ \\
2,4-dihydroxybenzoic acid & 4.67 & 1.93 & 2.97 & 0.31 & $\uparrow * *$ & $\downarrow * *$ \\
Kiwiionoside & 1.90 & 1.01 & 4.26 & 0.28 & $\uparrow * *$ & $\downarrow * *$ \\
11-hydroxyeicosatetraenoate glyceryl ester & 1.24 & 10.44 & 0.65 & 0.20 & $\downarrow * *$ & $\downarrow * *$ \\
Dictyoquinazol b & 1.11 & 1.57 & 1.73 & 0.21 & $\uparrow * *$ & $\downarrow * *$ \\
N-decanoylglycine & 2.01 & 2.64 & 3.18 & 0.41 & $\uparrow * *$ & $\downarrow * *$ \\
Mycotoxin t 2 & 7.19 & 1.19 & 5.26 & 0.32 & $\uparrow * *$ & $\downarrow * *$ \\
Ascorbyl palmitate & 1.13 & 1.53 & 0.66 & 1.66 & $\downarrow * *$ & $\uparrow * *$ \\
Met His Lys & 2.81 & 1.45 & 0.62 & 0.74 & $\downarrow * *$ & $\downarrow * *$ \\
(2-methyl-3-furyl)thio-2-butanone & 9.76 & 2.20 & 2.40 & 0.44 & $\uparrow * *$ & $\downarrow * *$ \\
Indoxyl sulfate & 9.50 & 1.63 & 0.51 & 2.02 & $\downarrow * *$ & $\uparrow * *$ \\
Glycochenodeoxycholic acid 3-glucuronide & 1.16 & 1.02 & 0.60 & 0.53 & $\downarrow * *$ & $\downarrow * *$ \\
MG(18:0/0:0/0:0) & 2.65 & 1.85 & 0.61 & 1.35 & $\downarrow * *$ & $\uparrow * *$ \\
\hline
\end{tabular}

Notes: VIP means variable importance in projection. FC refers to the fold change, and FC $>1$ or FC $<1$, indicated by up arrows or down arrows repectively, were usd to represent latively increased or decreased levels of the metabolites. ${ }^{* *}$ means $\mathrm{p}<0.01$ between groups.

\section{Metabolite profiling}

To better understand the effect of identified metabolites in serum and urine samples of rabbits of different groups, the enrichment of metabolic pathway was analyzed separately. Firstly, all the metabolites in each group, with FC $<0.67$ or FC $>1.5$, were selected and putted into MetaboAnalyst, which as a platform of comprehensive analysis for quantitative metabonomics data based on web. Secondly, the metabolites were matched according to the KEGG metabolic pathway database. Finally, the visualized results of metabolic pathway analysis are illustrated in Figure 5. As shown in Fig.5, the 
main metabolic pathways in serum samples disturbed by DON included: Glycerophospholipid metabolism, Tryptophan metabolism, Pentose and glucuronate interconversions, and Ether lipid metabolism, while their pathway impact were $22 \%, 14 \%$, $14 \%$ and $14 \%$ in order. At the same time, the main metabolic pathways in urine samples included: Caffeine metabolism, Glycine, serine and threonine metabolism, Terpenoid backbone biosynthesis, Pentose and glucuronate interconversions, while their pathway impact were $30 \%, 27 \%, 18 \%$ and $14 \%$ in order. Additionally, both the number of metabolic pathways and that of differential metabolites induced by DON in urine samples were higher than those in blood samples. In conclusion, the pathogenic mechanism of DON includes multiple levels, involving the metabolism of substances such as sugar, fat and protein, as well as their metabolic pathways, indicating that DON poisoning is caused by multiple factors acting on multiple links.

\section{Identified metabolites and disease induced by DON}

Metabolites in blood and urine are often the key to the diagnosis of diseases. In particular, metabolites in urine, which are the terminal metabolites in living organisms, are the most complex and most likely to reflect diseases in the body [30]. In order to further analysis the correlation between identified metabolites in serum and urine of rabbits induced by DON and specific diseases, the Pub Chem ID of the identified metabolites were uploaded into MetaboAnalyst platform and enrichment analysis were performed using package globaltest based on Quantitative enrichment analysis (QEA), which aimed to describe the correlation between compound concentration profiles and clinical outcomes. As a result, total of 22 kinds of diseases in serum samples and that of 65 kinds of diseases in urine 
were screened, which associated with the identified metabolites of rabbits interfered by DON (Table S4). In order to reveal the correlation between disease and identified metabolites inducted by DON for rabbits, the histograms have been drawn based on the top 14 associated diseases as the ordinate, and the number of associated metabolites as the abscissa (Fig.6). For serum samples, the associated diseases of the top three were Schizophrenia, Different seizure disorders, and Cirrhosis, with the number of the associated metabolites at 26, 24 and 23 in order. While in urine samples, associated diseases of the top three were Schizophrenia, Lung Cancer and Medium Chain Acyl-Coa Dehydrogenase Deficiency, with the number of the associated metabolites at 32, 32 and 28 in order. The correlating network diagram has been established via Cytoscape software, which aimed to further understand the correlation between specific diseases associated with identified metabolites affected by DON. It can be seen from Fig.7, for serum samples, Refractory localization-related epilepsy was correlated to the multiple diseases, while for urine samples, Short-chain 3-hydroxyacyl-coa Dehydrogenase deficiency, X-linked creatine-transporter defect Carbamoyl Phosphate Synthetase Deficiency and Sarcosinemia were correlated to the multiple diseases. The results of this study indicated that DON addition would cause changes of some metabolites related to specific diseases in the serum and urine of weaned rabbits. It is of great concern that the first related disease in both of serum and urine samples were schizophrenia, and multiple identified metabolites were associated with neurological diseases.

In recent years, numerous researches found that there exists a complex neuroendocrine network which connect the brain and gastrointestinal tract, so it is called "brain-gut axis" 
[31]. ${ }^{1}$ As a two-way pathway between the central nervous system and the gastrointestinal tract, abnormal signal transduction is associated with numerous diseases, such as inflammatory, functional gastrointestinal diseases and feeding disorders, and so on [32]. DON can act on the central nervous system through quickly the blood-brain barrier, cause changes in certain chemicals, and then lead to eating less or refusing food $[33,34]$. Consequently, it is need to further investigate whether chronically low intake of DON is a risk factor for psychiatric disorders. A growing number of studies have demonstrated that DON can induce changes in intestinal microbialflora, whose metabolites are important mediators linking the intestinal tract and the brain-gut-axis [35]. It also have been shown that the disorder of intestinal flora are related to neurological diseases such as depression [36]. Therefore, the next step on the researchs of the relationship among DON, metabolites, intestinal flora and neuromodulation in vivo will help us to further understand the pathogenic mechanism of DON, which beneficial to prevent and control DON contamination.

\section{Additional files}

Additional file 1. Table S1. The elution gradient of HPLC in this experiment; Table S2. The parameters of mass spectrum in this experiment. (DOC, $41 \mathrm{~kb}$ )

Additional file 2. Table S3. Differential metabolites in serum and urine. (XLS, 493kb)

Additional file 3. Table S4. Associated diseases and metabolites in serum and urine. (XLS, $13 \mathrm{~kb})$ 
411 DON: deoxynivalenol; DOM: de-epoxidation deoxynivalenol; PCA: principal component analysis; OPLS-DA: orthogonal partial least-squares discriminant analysis; VIP: variable

413 important in projection; FC: fold change; RPT: 200 response permutation testing; LC-MS:

414 Liquid chromatography-tandem mass spectrometry;

\section{Acknowledgement}

416 We would like to thank our anonymous reviewers and our colleagues from the Animal

417 Nutrition Laboratory for their valuable critiques and suggestions.

\section{$418 \quad$ Funding}

This work was supported by the Modern Agro-industry Technology Research system

(CARS-43-B-1), Natural Science Foundation of Shandong Province (ZR2018QC004, (2019GNC106007) and Funds from the Shandong 'Double Tops' Program and Youth Science.

\section{Availability of data and materials}

The datasets used and/or analysed during the current study are available from the corresponding author on reasonable request.

\section{Authors' contributions}

Li F.C. designed the study. Huang L.B. performed the experiments and wrote a draft of the paper. Liu Q.C. performed animal trial. Wang P.W. analyzed the data. Chen H.J. experiments and revised the paper. 
433 The experimental protocol concerning live animals in this study has been ratified by the 434 Animal Protection Committee of Shandong Agricultural University (Certification No. 435 ACSA-2019-0025), and the operation procedures are in compliance with the ethical 436 regulations of laboratory animals promulgated by the Ministry of Science and 437 Technology of China.

\section{Consent for publication}

Not applicable.

\section{Competing interests}

441 The authors declare no conflict of interest.

\section{Author details}

\section{References}

449 1. Lee HJ, Ryu D. Worldwide Occurrence of Mycotoxins in Cereals and Cereal-Derived Chem. 2017; 65(33): 7034-51. https://doi.org/10.1021/acs.jafc.6b04847

2. Bryla M, Waskiewicz A, Ksieniewicz-Wozniak E, Szymczyk K, Jedrzejczak R. 
Occurrence, and Toxicity: An Updated Review. Molecules. 2018; 23: 963. https://doi.org/10.3390/molecules23040963

3. Liao Y, Peng Z, Chen L, Nussler AK, Liu L, Yang W. Deoxynivalenol, gut microbiota and immunotoxicity: A potential approach? Food Chem Toxicol. 2018; 112: 342-54. https://doi.org/10.1016/j.fct.2018.01.013

4. Cimbalo A, Alonso-Garrido M, Font G, Manyes L. Toxicity of mycotoxins in vivo on vertebrate organisms: A review. Food. Chem. Toxicol. 2020; 137: 111161. https://doi.org/10.1016/j.fct.2020.111161

5. Ghareeb K, Awad WA, Bohm J, Zebeli Q. Impacts of the feed contaminant deoxynivalenol on the intestine of monogastric animals: poultry and swine. J. Appl Toxicol. 2015; 35 (4): 327-37. https://doi.org/10.1002/jat.3083

6. Pestka JJ, Islam Z, Amuzie CJ. Immunochemical assessment of deoxynivalenol tissue distribution following oral exposure in the mouse. Toxicol. Lett. 2008; 178 (2): 83-87. https://doi.org/10.1016/j.toxlet.2008.02.005

7. Wan D, Huang L, Pan Y, Wu Q, Chen D, Tao Y, Wang X, Liu Z, Li J, Wang L, Yuan Z. Metabolism, Distribution, and Excretion of Deoxynivalenol with Combined Techniques of Radiotracing, High-Performance Liquid Chromatography Ion Trap Time-of-Flight Mass Spectrometry, and Online Radiometric Detection. J.Agric.Food Chem. 2014; 62(1): 288-96. https://doi.org/10.1021/jf4047946

8. Warth B, Sulyok M, Fruhmann P, Mikula H, Berthiller F, Schuhmacher R, Hametner C, Abia WA, Adam G, Frohlich J, Krska R. Development and validation of a rapid multi-biomarker liquid chromatography/tandem mass spectrometry method to 
assess human exposure to mycotoxins. Rapid. Commun.Mass.Spectrom. 2012; 26(13): 1533-40. https://doi.org/10.1002/rcm.6255

9. Wu Q, Dohnal V, Huang L, Kuča K, Yuan Z. Metabolic pathways of trichothecenes. Drug Metab.Rev. 2010; 42 (2): 250-67. https://doi.org/10.1021/jf4047946

10. Schwartz-Zimmermann HE, Fruhmann P, Danicke S, Wiesenberger G, Caha S,

12. Danicke S, Valenta H, Ganter M, Brosig B, Kersten S, Diesing AK, Kahlert S, Panther P, Kluess J, Rothkotter HJ. Lipopolysaccharides (LPS) modulate the metabolism of deoxynivalenol (DON) in the pig. Mycotoxin Res. 2014; 30 (3): 161-70. https://doi.org/10.1007/s12550-014-0201-7

13. Schwartz-Zimmermann HE, Hametner C, Nagl V, Fiby I, Macheiner L, Winkler J, Danicke S, Clark E, Pestka JJ, Berthiller F. Glucuronidation of deoxynivalenol (DON) by different animal species: identification of iso-DON glucuronides and iso-deepoxy-DON glucuronides as novel DON metabolites in pigs, rats, mice, and cows. Arch. Toxicol. 2017; 91(12): 3857-72. https:/doi.org/10.1007/s00204-017-2012-z 
14. Li F, Wang J, Huang L, Chen H, Wang C. Effects of Adding Clostridium sp. WJ06 on Intestinal Morphology and Microbial Diversity of Growing Pigs Fed with Natural Deoxynivalenol Contaminated Wheat. Toxins. 2017; 9: 83. https://doi.org/10.3390/toxins9120383

15. Wu L, W WC, Huang R, Cui Z, He L, Y J, Duan J, Li T, Wang JQ. Deoxynivalenol residues in edible tissue of infested pig. J. Food. Agric. Environ. 2013;11:1129-33.

16. Gratz SW, Currie V, Duncan G, Jackson D. Multimycotoxin Exposure Assessment in UK Children Using Urinary Biomarkers-A Pilot Survey. J. Agric. Food Chem. 2020; 68 (1): 351-57. https://doi.org/10.1021/acs.jafc.9b03964

17. Hopton RP, Turner E, Burley VJ, Turner PC, Fisher J. Urine metabolite analysis as a function of deoxynivalenol exposure: an NMR-based metabolomics investigation. Food Addit.Contam. 2010; 27(2):255-61. https://doi.org/10.1080/19440040903314015

18. Xu Y, Ji J, Wu H, Pi F, Blazenovic I, Zhang Y, Sun X. Untargeted GC-TOFMS-based cellular metabolism analysis to evaluate ozone degradation effect of deoxynivalenol. Toxicon. 2019; 168:49-57. https://doi.org/10.1016/j.toxicon.2019.06.022

19. Saia S, Fragasso M, De Vita P, Beleggia R. Metabolomics Provides Valuable Insight for the Study of Durum Wheat: A Review. J. Agric. Food Chem. 2019; 67(11): 3069-85. https://doi.org/10.1021/acs.jafc.8b07097

20. He H, An F, Huang Q, Kong Y, He D, Chen L, Song H. Metabolic effect of AOS-iron in rats with iron deficiency anemia using LC-MS/MS based metabolomics. Food. Res. Int. 2020; 130. https://doi.org/10.1016/j.foodres.2019.108913 
21. Greco MV, Pardo AG, Ludemann V, Martino PE, Pose GN. Mycoflora and natural incidence of selected mycotoxins in rabbit and Chinchilla feeds. Sci. World. J. 2012; 2012: 956056. https://doi.org/10.1100/2012/956056

22. Zhu Y, Wang C, Li F. Impact of dietary fiber/starch ratio in shaping caecal microbiota in rabbits. Can J.Microbiol. 2015;61(10): 771-84. https://doi.org/10.1139/cjm-2015-0201

23. Wang C, Zhu Y, Li F, Huang L. The Effect of Lactobacillus isolates on growth performance, immune response, intestinal bacterial community composition of growing Rex Rabbits. J. Anim Physiol. Anim Nutr. 2017; 101(5): e1-e9. https://doi.org/e1-e13.10.1111/jpn.12629

24. Brezina U, Valenta H, Rempe I, Kersten S, Humpf HU, Danicke S. Development of a liquid chromatography tandem mass spectrometry method for the simultaneous determination of zearalenone, deoxynivalenol and their metabolites in pig serum. Mycotoxin. Res. 2014; 30 (3):171-86. https://doi.org/10.1007/s12550-014-0200-8

25. Barnett AM, Roy NC, McNabb WC, Cookson AL. The interactions between endogenous bacteria, dietary components and the mucus layer of the large bowel. Food Funct. 2012; 3(7): 690-9. https://doi.org/10.1039/c2fo30017f

26. Rotter BA, Prelusky DB, Pestks JJ. Toxicology of deoxynivalenol (vomitoxin). J. Toxicol. Environ. Health. 1996; 48:1-34. https://doi.org/10.1080/713851046

27. Frey JC, Pell AN, Berthiaume R, Lapierre H, Lee S, Ha JK, Mendell JE, Angert ER. Comparative studies of microbial populations in the rumen, duodenum, ileum and faeces of lactating dairy cows. J. Appl. Microbiol. 2010; 108 (6):1982-93. https://doi.org/10.1111/j.1365-2672.2009.04602.x 
28. Ji C, Fan Y, Zhao L. Review on biological degradation of mycotoxins. Anim. Nutr. 2016; 2(3): 127-33. https://doi.org/10.1016/j.aninu.2016.07.003

29. Lessard M, Savard C, Deschene K, Lauzon K, Pinilla VA, Gagnon CA, Lapointe J, Guay F, Chorfi Y. Impact of deoxynivalenol (DON) contaminated feed on intestinal integrity and immune response in swine. Food. Chem. Toxicol. 2015; 80: 7-16. https://doi.org/10.1016/j.fct.2015.02.013

30. Fernandez-Ochoa A, Quirantes-Pine, R, Borras-Linares I, Gemperline D, Consortium PC, Alarcon Riquelme ME, Beretta L, Segura-Carretero A. Urinary and plasma metabolite differences detected by HPLC-ESI-QTOF-MS in systemic sclerosis patients. J. Pharm. Biomed. Anal. 2019; 162: 82-90. https://doi.org/10.1016/j.jpba.2018.09.021

31. Clemmensen C, Muller TD, Woods SC, Berthoud HR, Seeley RJ, Tschop MH. Gut-Brain Cross-Talk in Metabolic Control. Cell. 2017; 168 (5): 758-74. https://doi.org/10.1016/j.cell.2017.01.025

32. Labanski A, Langhorst J, Engler H, Elsenbruch S. Stress and the brain-gut axis in functional and chronic-inflammatory gastrointestinal diseases: A transdisciplinary challenge. Psychoneuroendocrino. 2020; 111: 104501. https://doi.org/10.1016/j.psyneuen.2019.104501

33. Wang X, Chen X, Cao L, Zhu L, Zhang Y, Chu X, Zhu D, Rahman SU, Peng C, Feng S, Li Y, Wu J. Mechanism of deoxynivalenol-induced neurotoxicity in weaned piglets is linked to lipid peroxidation, dampened neurotransmitter levels, and interference 
with calcium signaling. Ecotoxicol. Environ. Saf. 2020; 194:110382.

https:/doi.org/10.1016/j.ecoenv.2020.110382

563

34. Miro-Abella E, Torrell H, Herrero P, Canela N, Arola L, Borrull F, Ras R, Fontanals N. Monitoring and evaluation of the interaction between deoxynivalenol and gut microbiota in Wistar rats by mass spectrometry-based metabolomics and next-generation sequencing. Food. Chem. Toxicol. 2018; 121: 124-130. https://doi.org/10.1016/j.fct.2018.08.006

35. Long-Smith C, O'Riordan KJ, Clarke G, Stanton C, Dinan TG, Cryan JF. Microbiota-Gut-Brain Axis: New Therapeutic Opportunities. Annu. Rev. Pharmacol. Toxicol. 2020; 60: 477-502. https://doi.org/10.1146/annurev-pharmtox-010919-023628

36. Peng Z, Chen L, Xiao J, Zhou X, Nussler AK, Liu L, Liu J, Yang W. Review of mechanisms of deoxynivalenol-induced anorexia: The role of gut microbiota. J. Appl.Toxicol. 2017; 37(9): 1021-29. https://doi.org/10.1002/jat.3475 

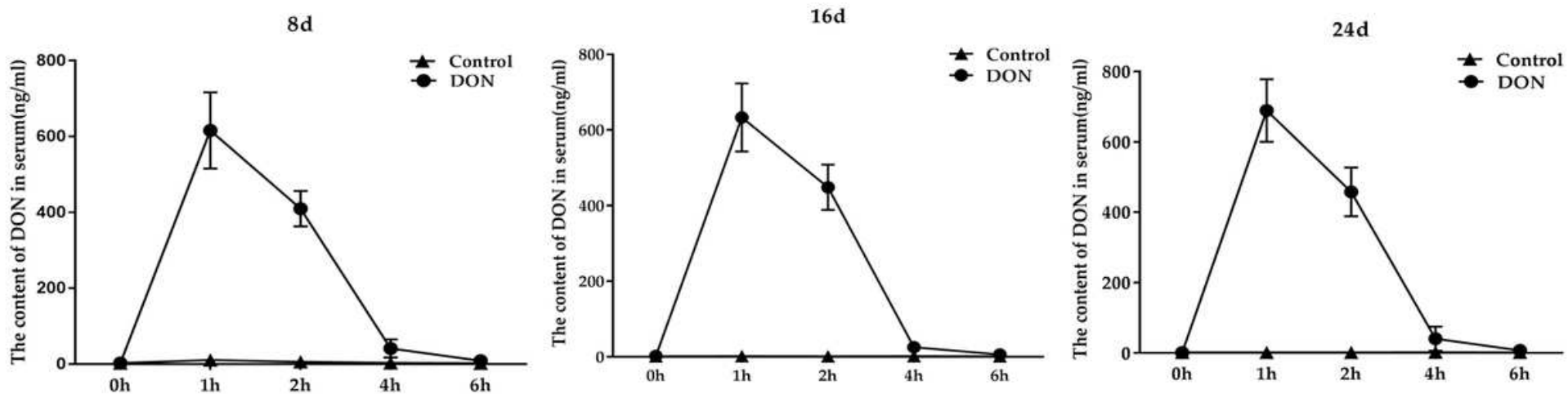

Figure 1

The concent of $D O N$ in serum samples $(M \pm S D, n=8) .8 d, 16 d$ and $24 d$ refers to the day of $D O N$ exposure. $x$-axis refers to different blood sampling times at same trial day. Control and DON refers to different group.
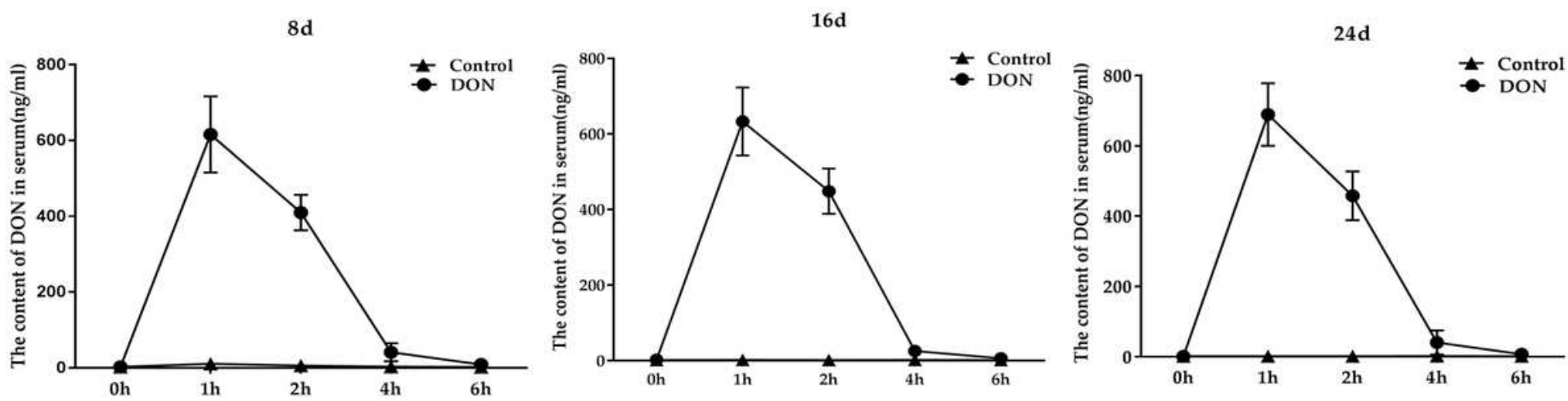

Figure 1

The concent of DON in serum samples $(M \pm S D, n=8) .8 d, 16 d$ and $24 d$ refers to the day of DON exposure. $\mathrm{x}$-axis refers to different blood sampling times at same trial day. Control and DON refers to different group.
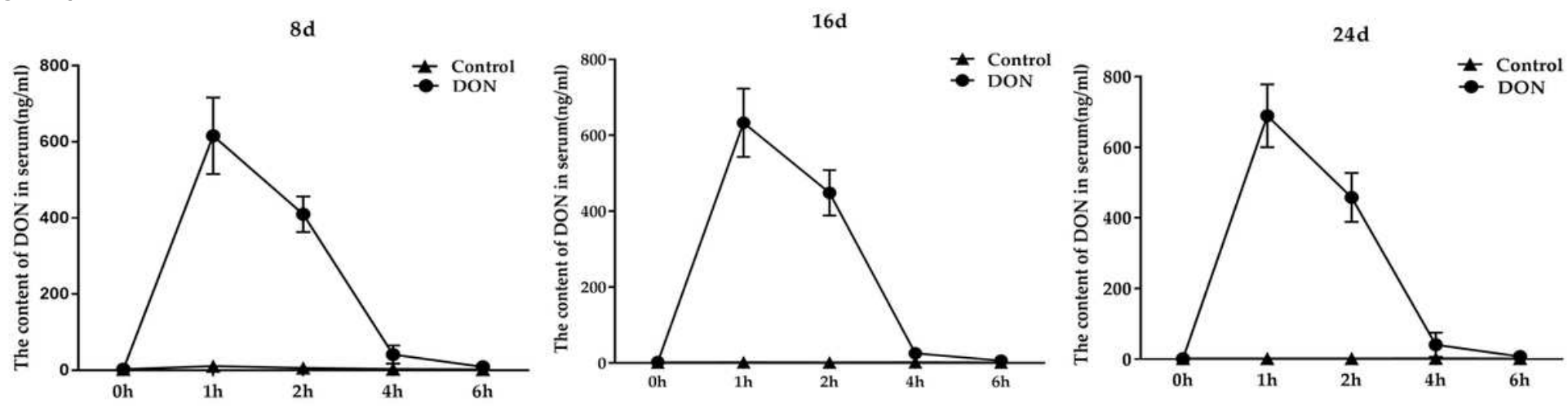

Figure 1 
The concent of DON in serum samples $(M \pm S D, n=8) .8 d, 16 d$ and $24 d$ refers to the day of DON exposure. $\mathrm{x}$-axis refers to different blood sampling times at same trial day. Control and DON refers to different group.
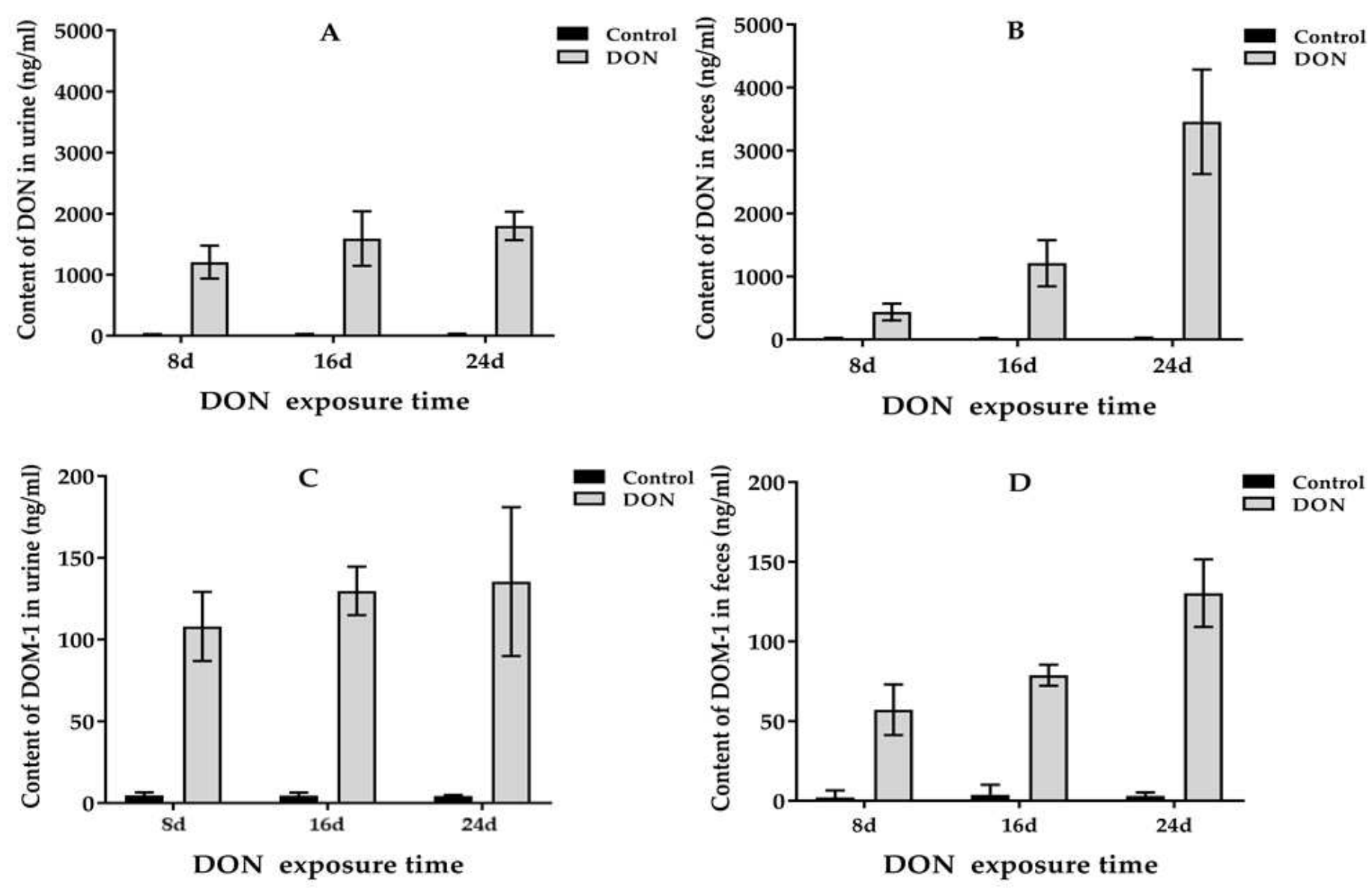

Figure 2

The concentration DON and DOM-1 in urine $(A, C)$ and feces $(B, D)$ samples at differnet period of DON exposure (Mean $\pm S D, n=8)$. Control and DON refers to different group. 

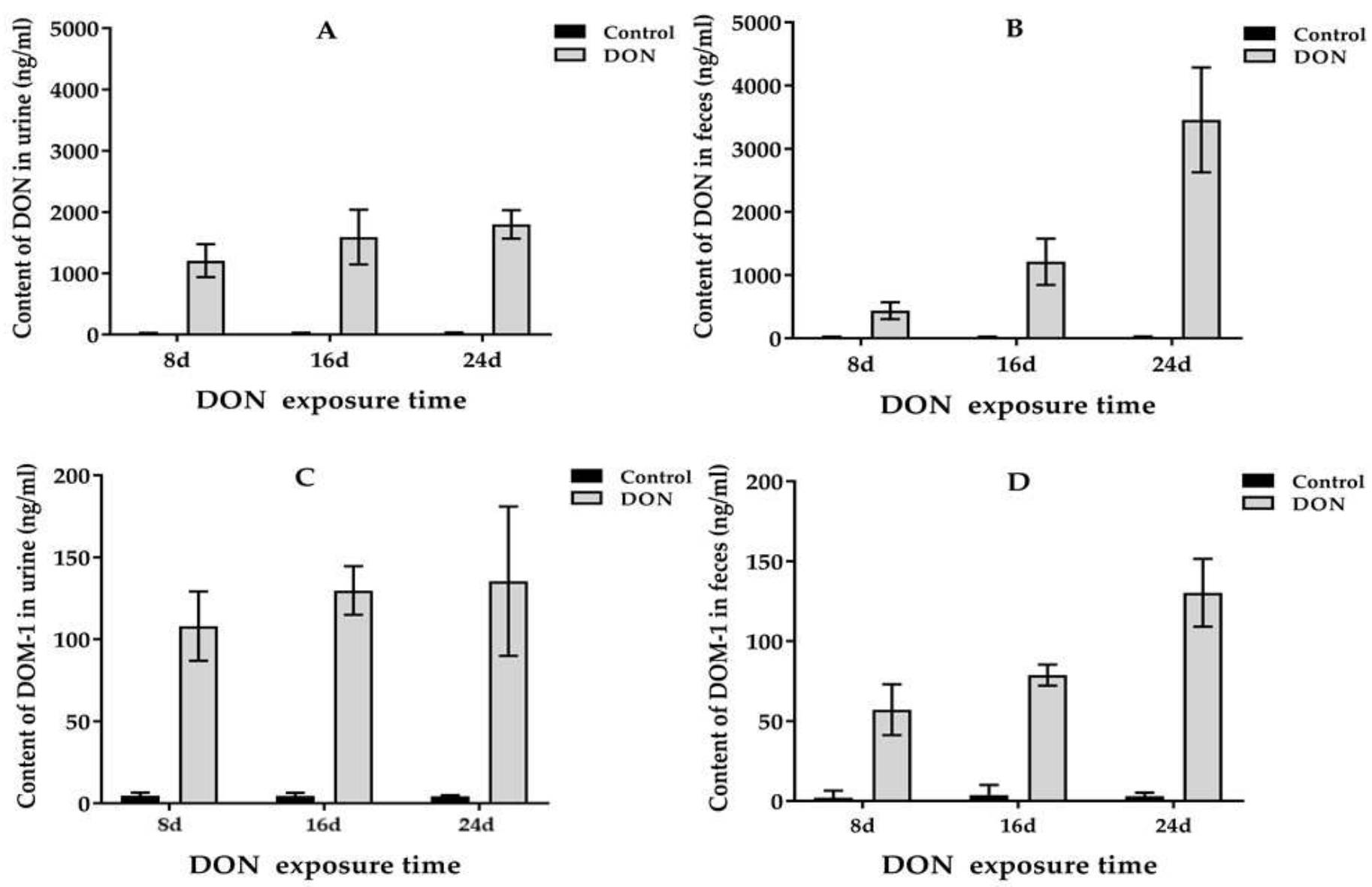

Figure 2

The concentration DON and DOM-1 in urine $(A, C)$ and feces $(B, D)$ samples at differnet period of DON exposure (Mean $\pm S D, n=8)$. Control and DON refers to different group. 

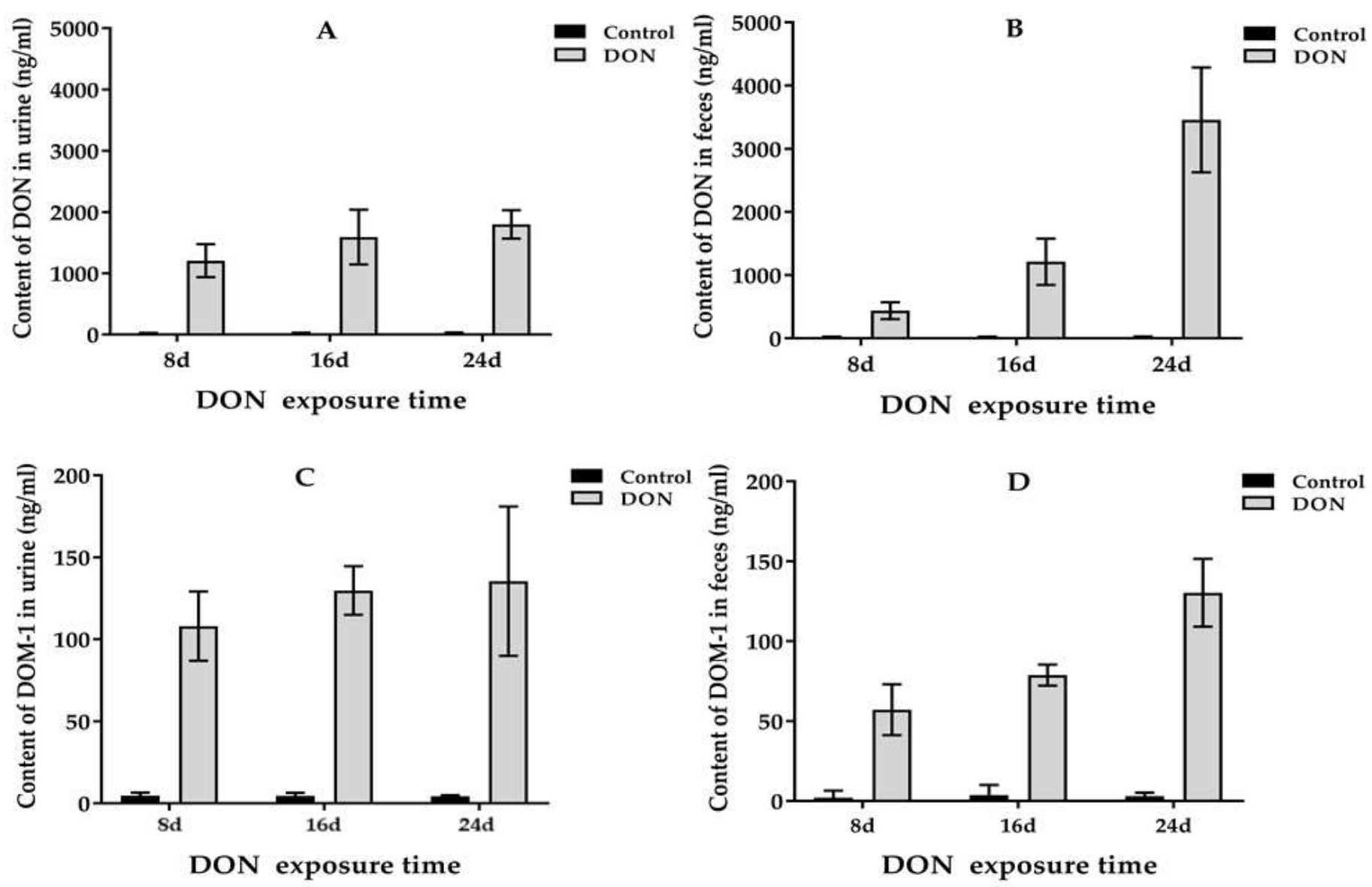

Figure 2

The concentration DON and DOM-1 in urine $(A, C)$ and feces $(B, D)$ samples at differnet period of DON exposure (Mean $\pm S D, n=8)$. Control and DON refers to different group. 


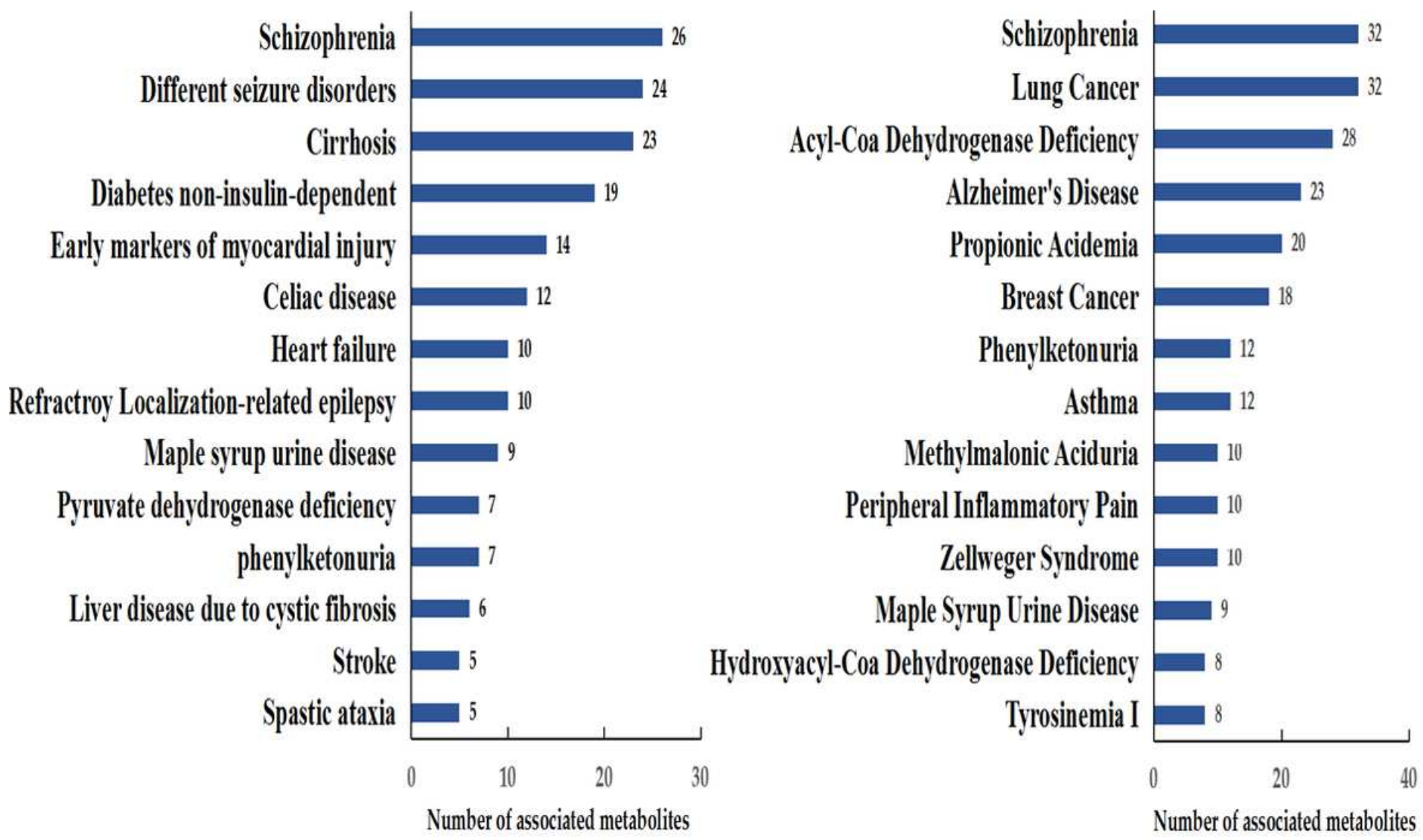

\section{Figure 3}

Multivariate analysis of serum (left) and urine (right) metabolites between two groups.A,B refers to PCA score plots, C,D refers to OPLS-DA score plots, and E,F represent the result of RPT. 


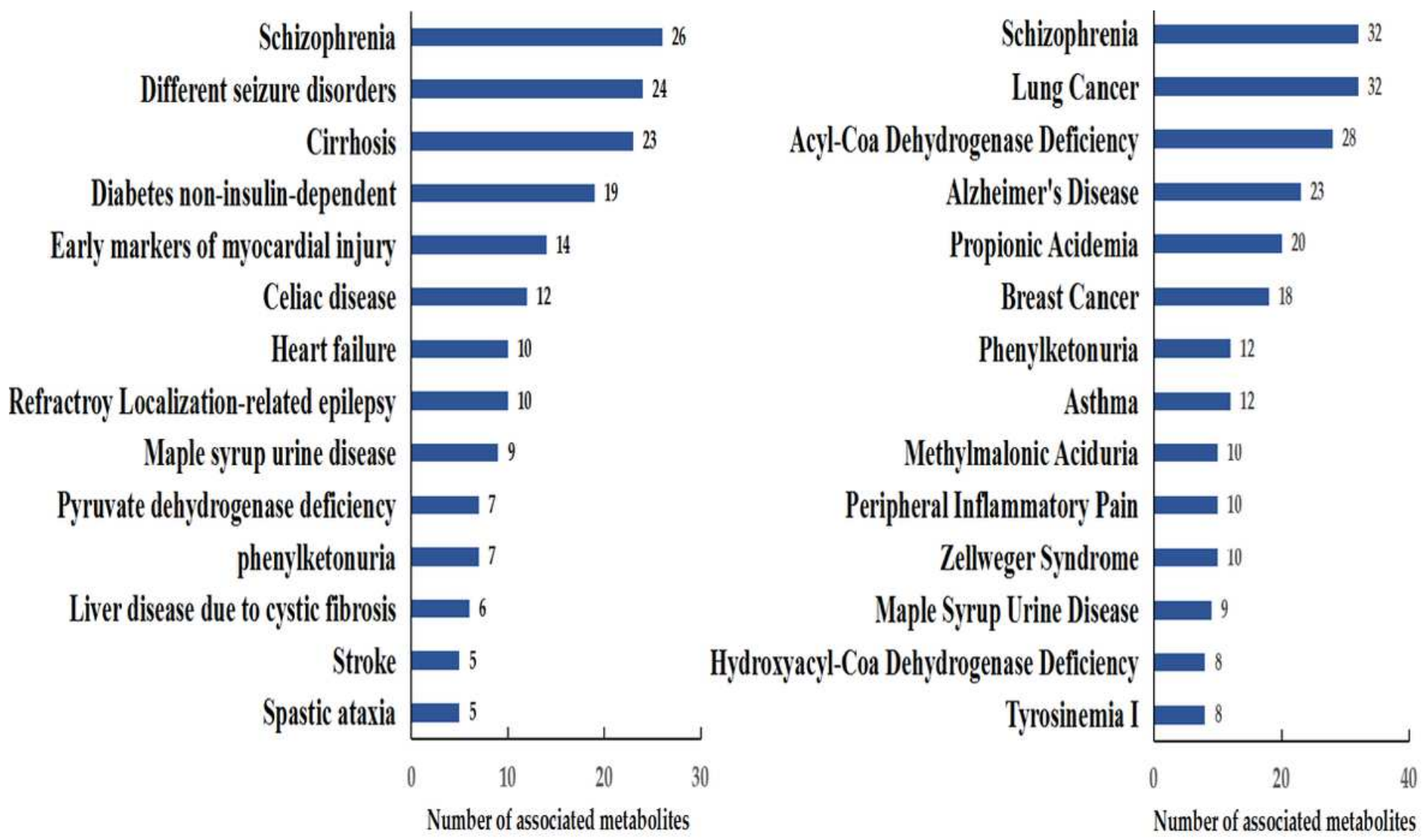

\section{Figure 3}

Multivariate analysis of serum (left) and urine (right) metabolites between two groups.A,B refers to PCA score plots, C,D refers to OPLS-DA score plots, and E,F represent the result of RPT. 


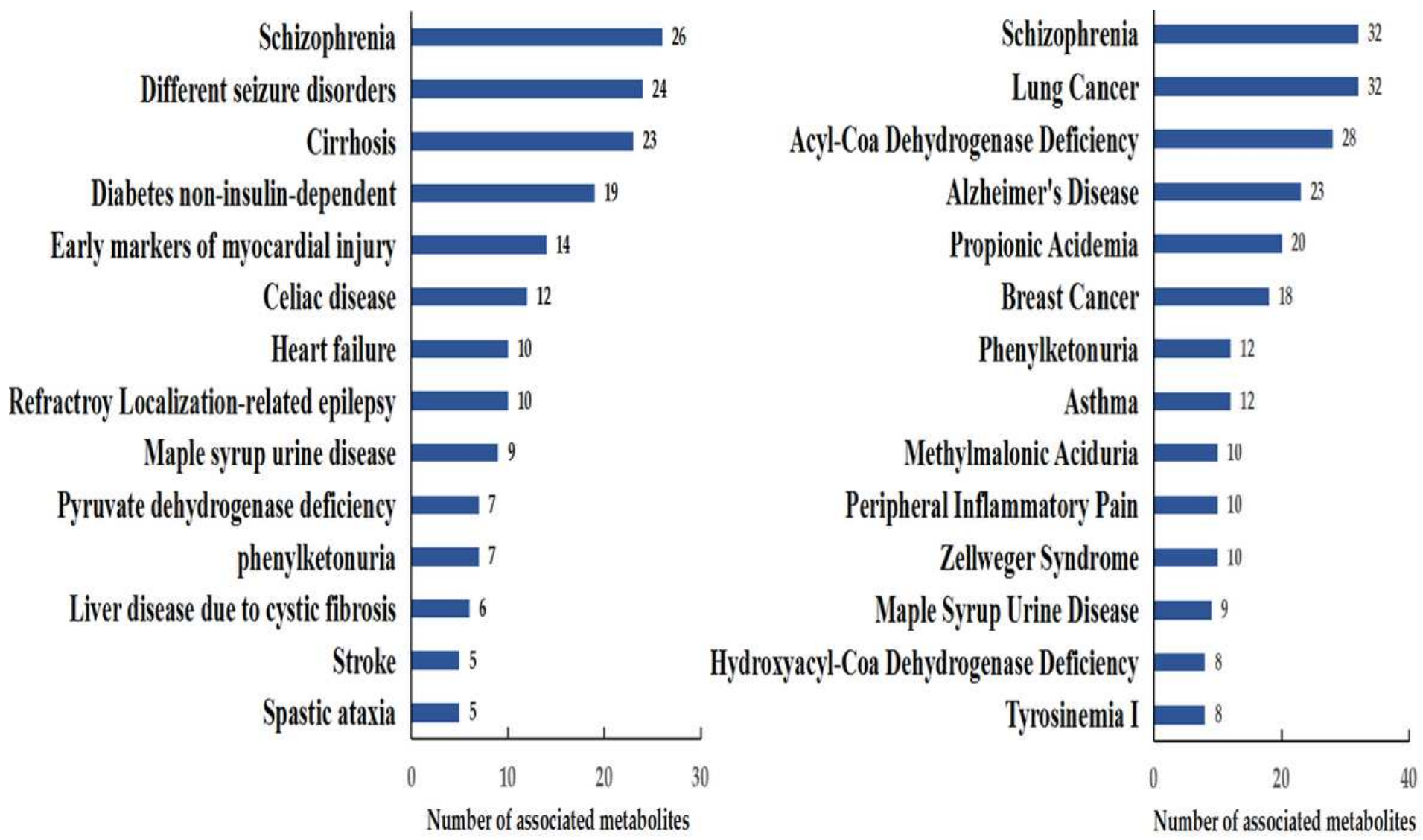

\section{Figure 3}

Multivariate analysis of serum (left) and urine (right) metabolites between two groups.A,B refers to PCA score plots, C,D refers to OPLS-DA score plots, and E,F represent the result of RPT. 

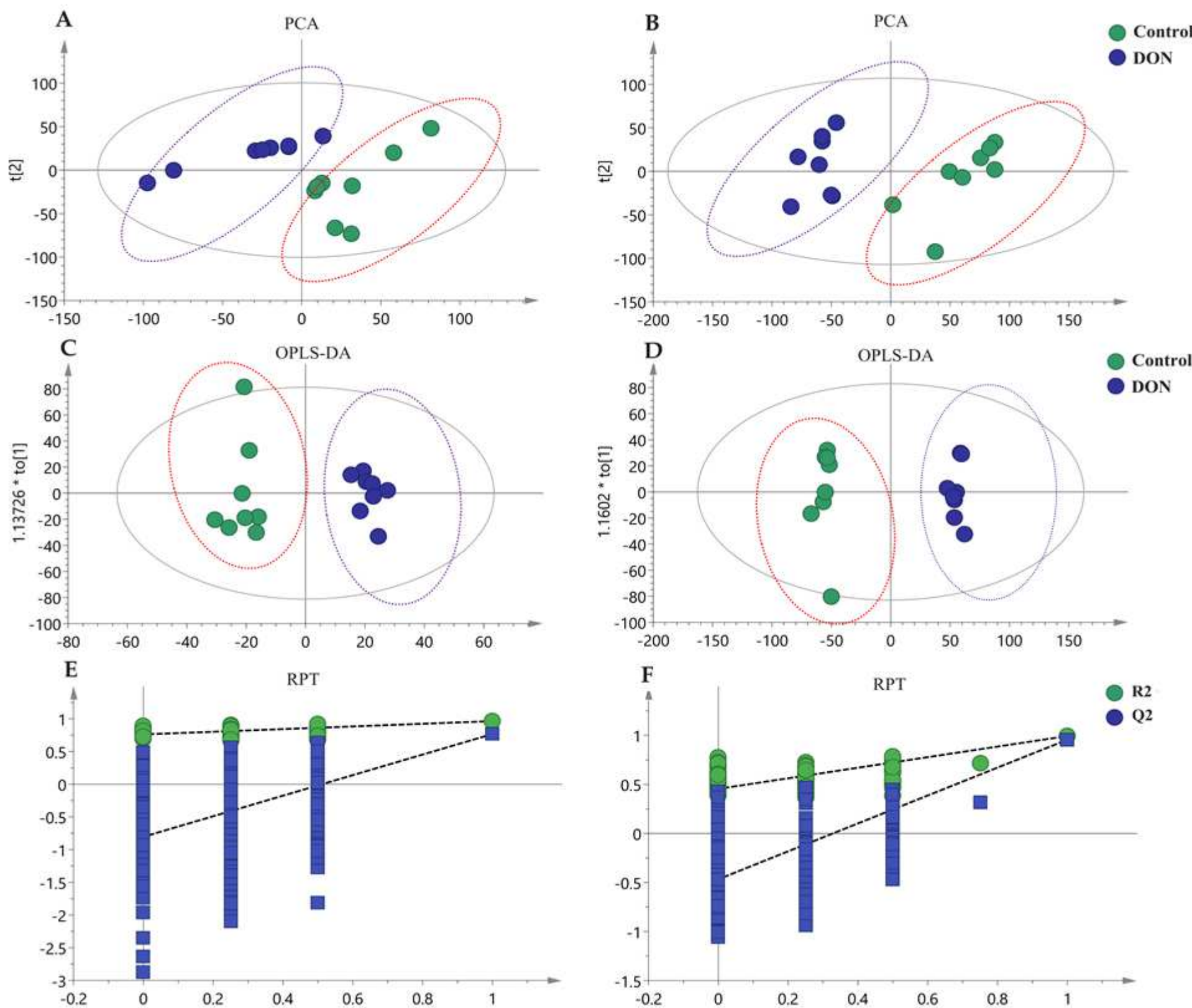

Figure 4

Differential metabolites analysis of serum and urine by venn method 

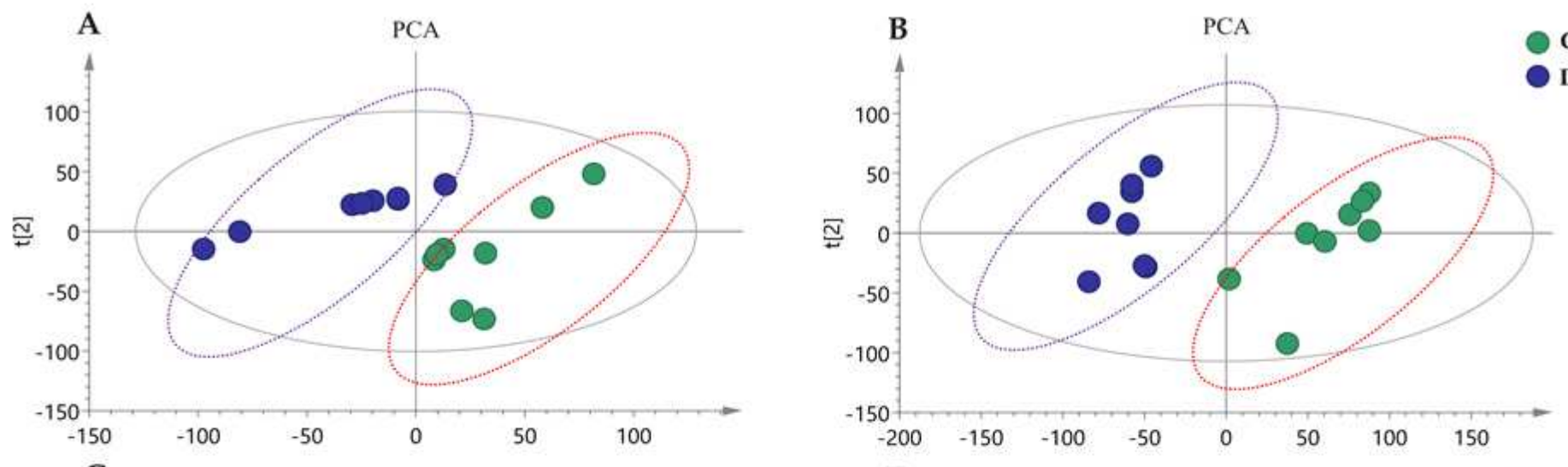

Control

DON
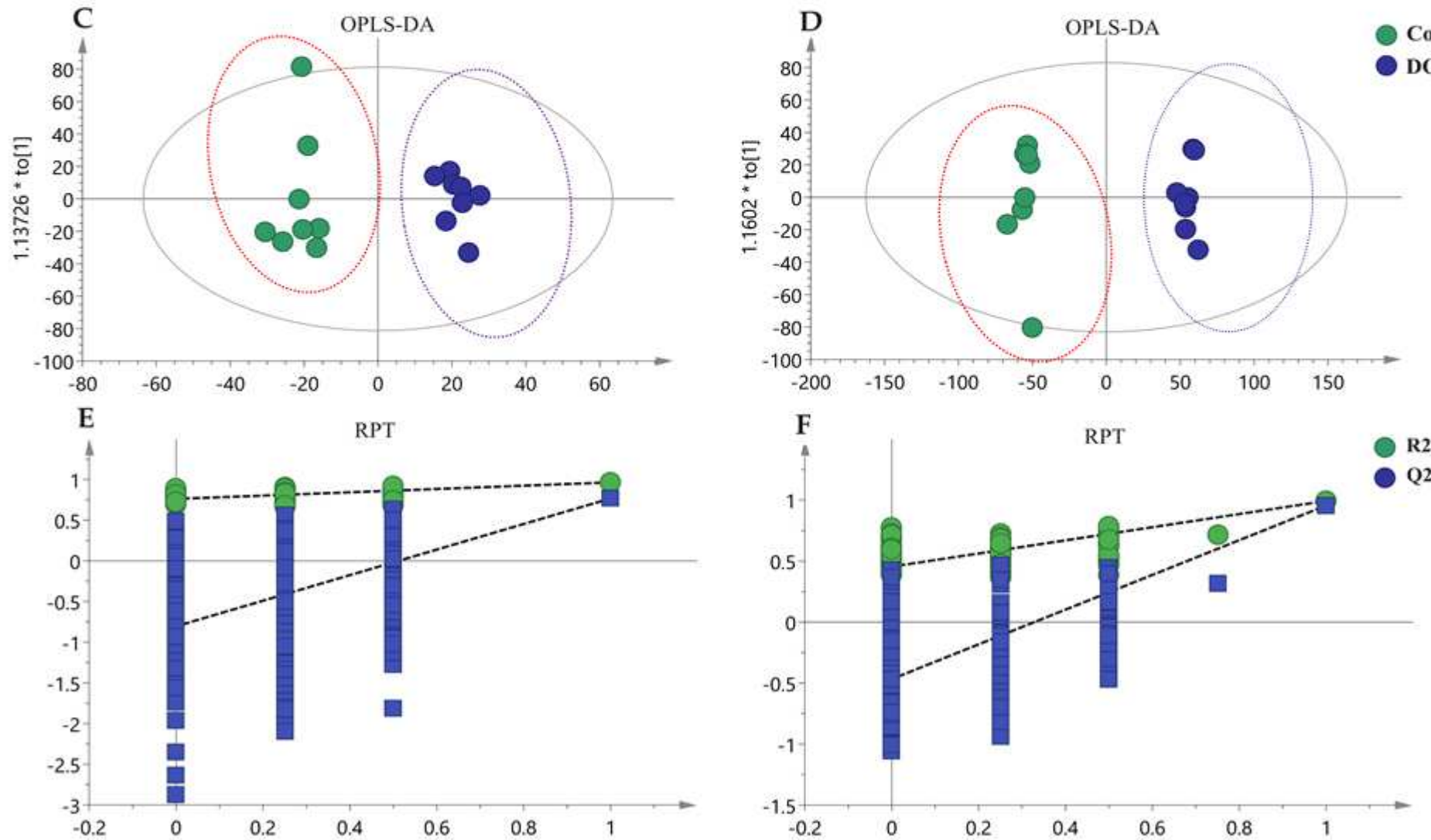

Figure 4

Differential metabolites analysis of serum and urine by venn method 

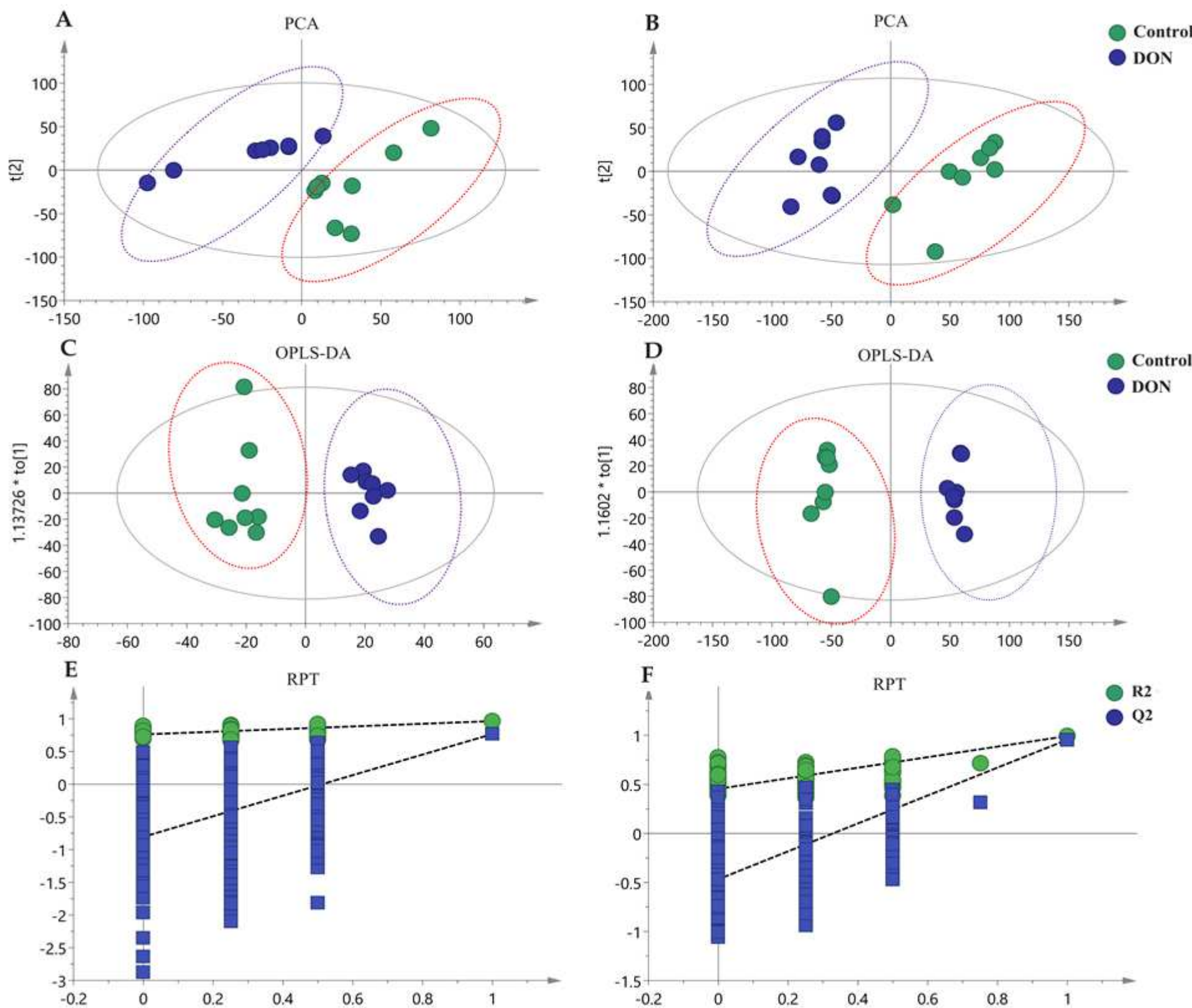

Figure 4

Differential metabolites analysis of serum and urine by venn method 

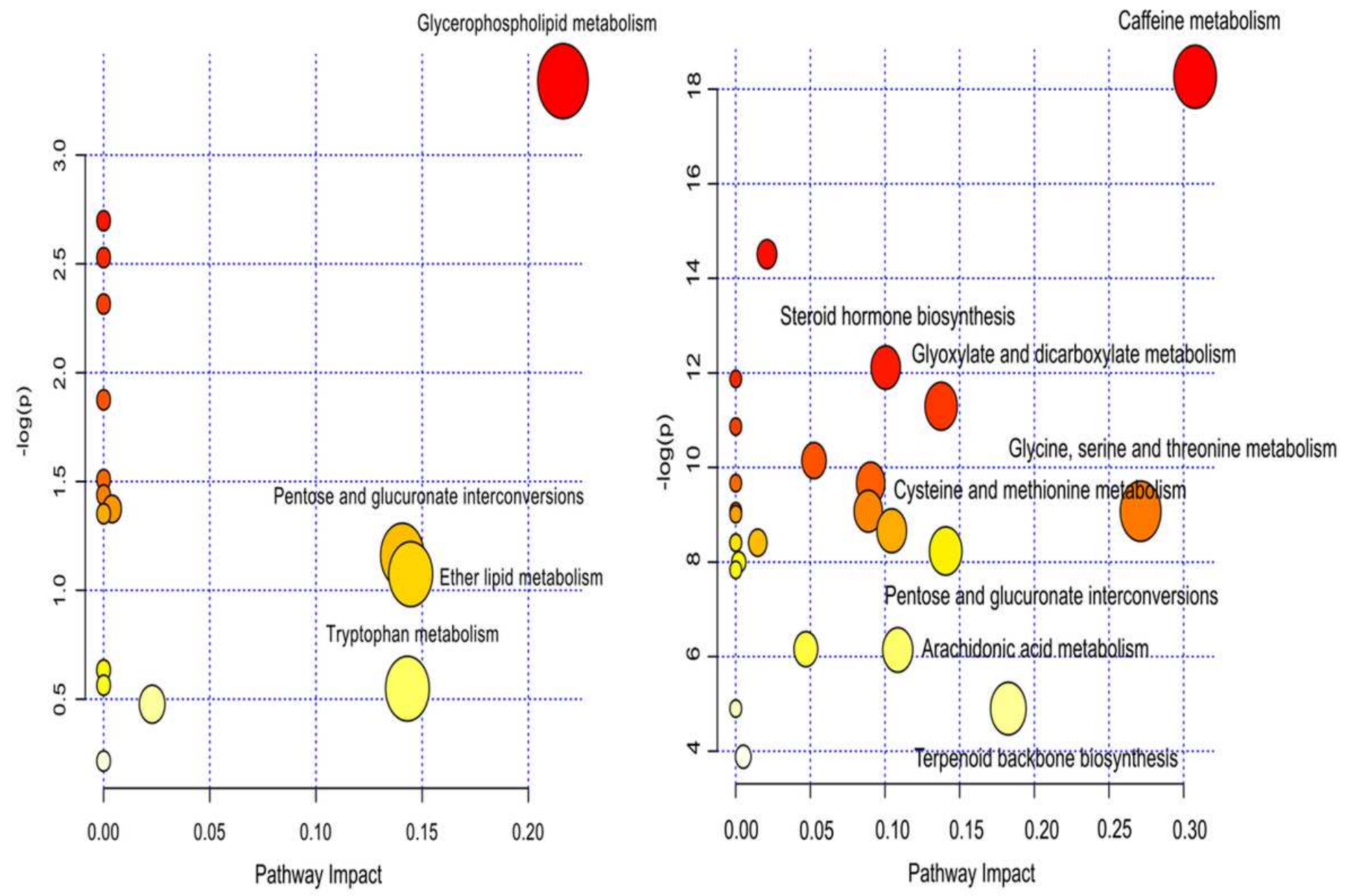

\section{Figure 5}

The pathway analysis of the identified metabolites in serum (left) and urine (right) of rabbits. Selected metabolites based on FC $<0.67$ or $>1.50$. X-axis represents the pathway impact and $y$-axis represents the pathway enrichment. Larger sizes and darker colors represent higher pathway enrichment and higher pathway impact values. 

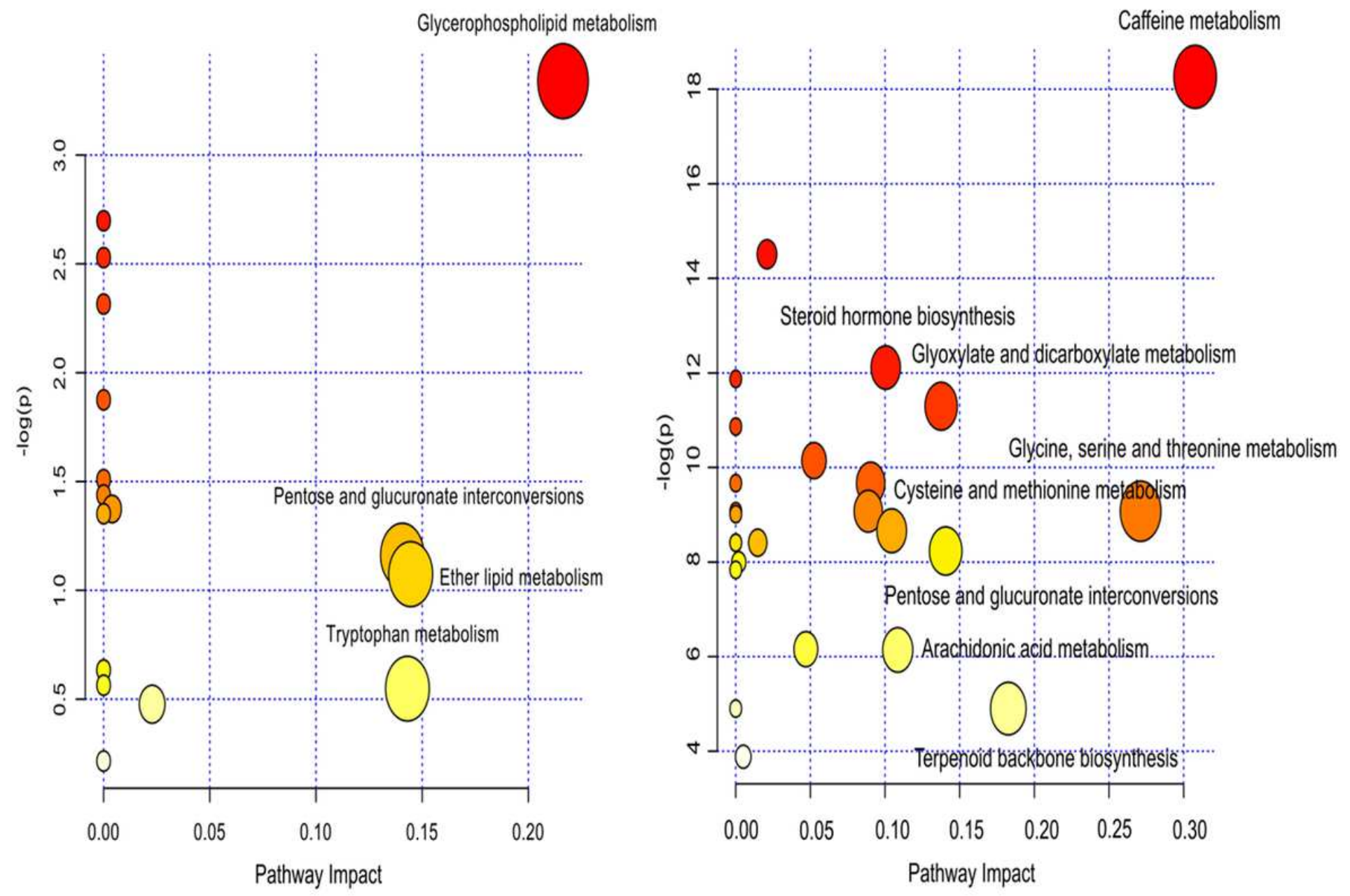

\section{Figure 5}

The pathway analysis of the identified metabolites in serum (left) and urine (right) of rabbits. Selected metabolites based on FC $<0.67$ or $>1.50$. X-axis represents the pathway impact and $y$-axis represents the pathway enrichment. Larger sizes and darker colors represent higher pathway enrichment and higher pathway impact values. 

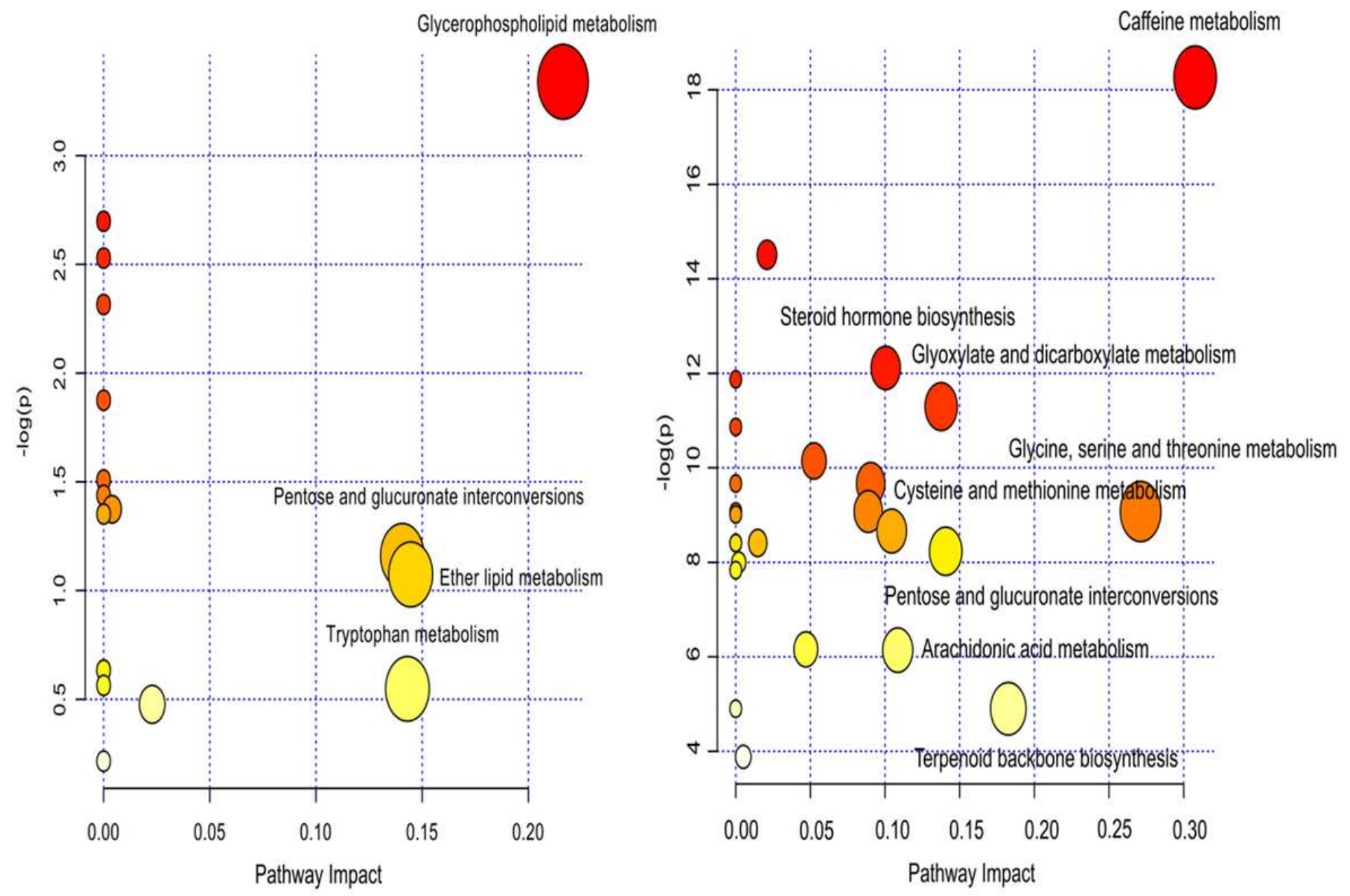

\section{Figure 5}

The pathway analysis of the identified metabolites in serum (left) and urine (right) of rabbits. Selected metabolites based on FC $<0.67$ or $>1.50$. X-axis represents the pathway impact and $y$-axis represents the pathway enrichment. Larger sizes and darker colors represent higher pathway enrichment and higher pathway impact values. 


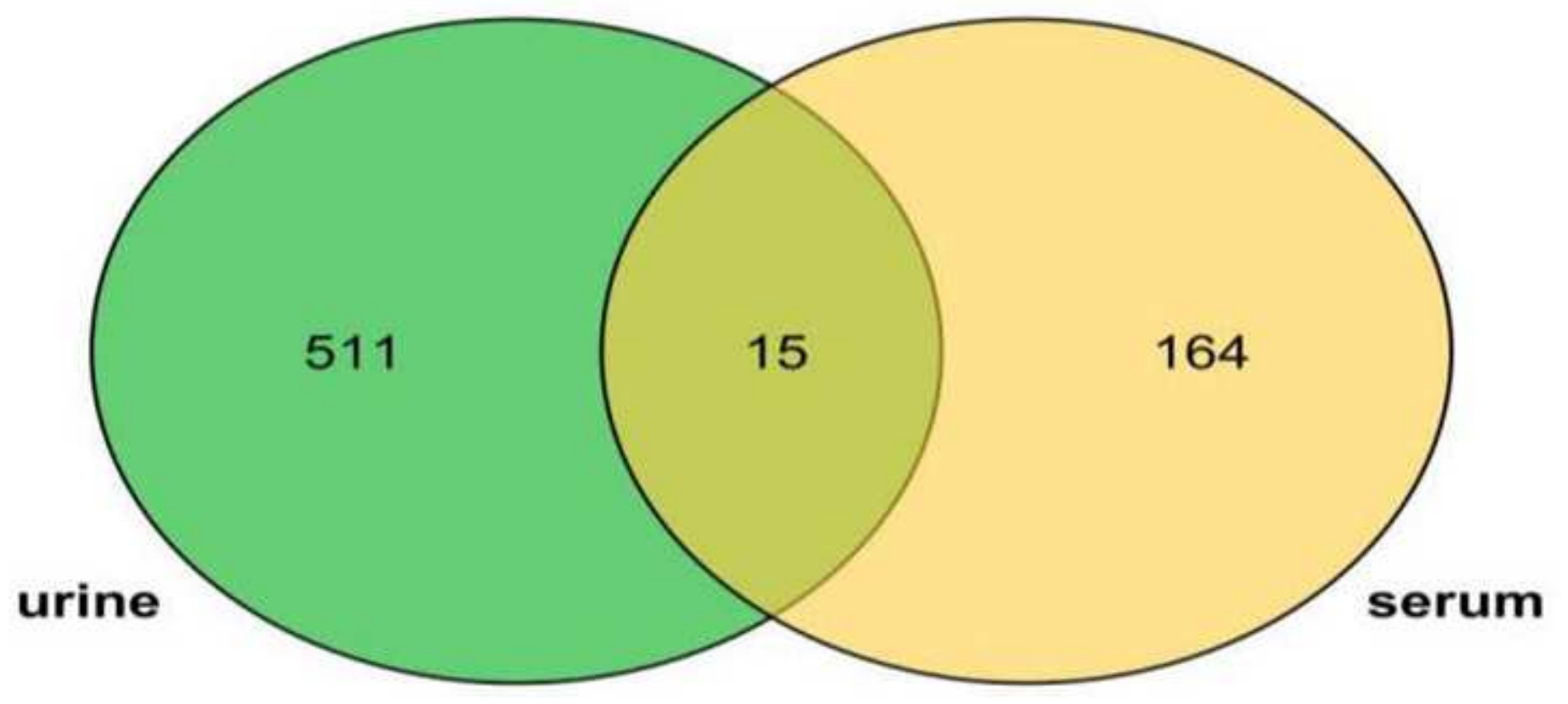

\section{Figure 6}

The bar graph of the correlation of spicific diseases and associated metabolites in serum (left) and urine (right). X-axis represents the number of assocated metabolites and $y$-axis represents the spicific diseases profiled by QEA.

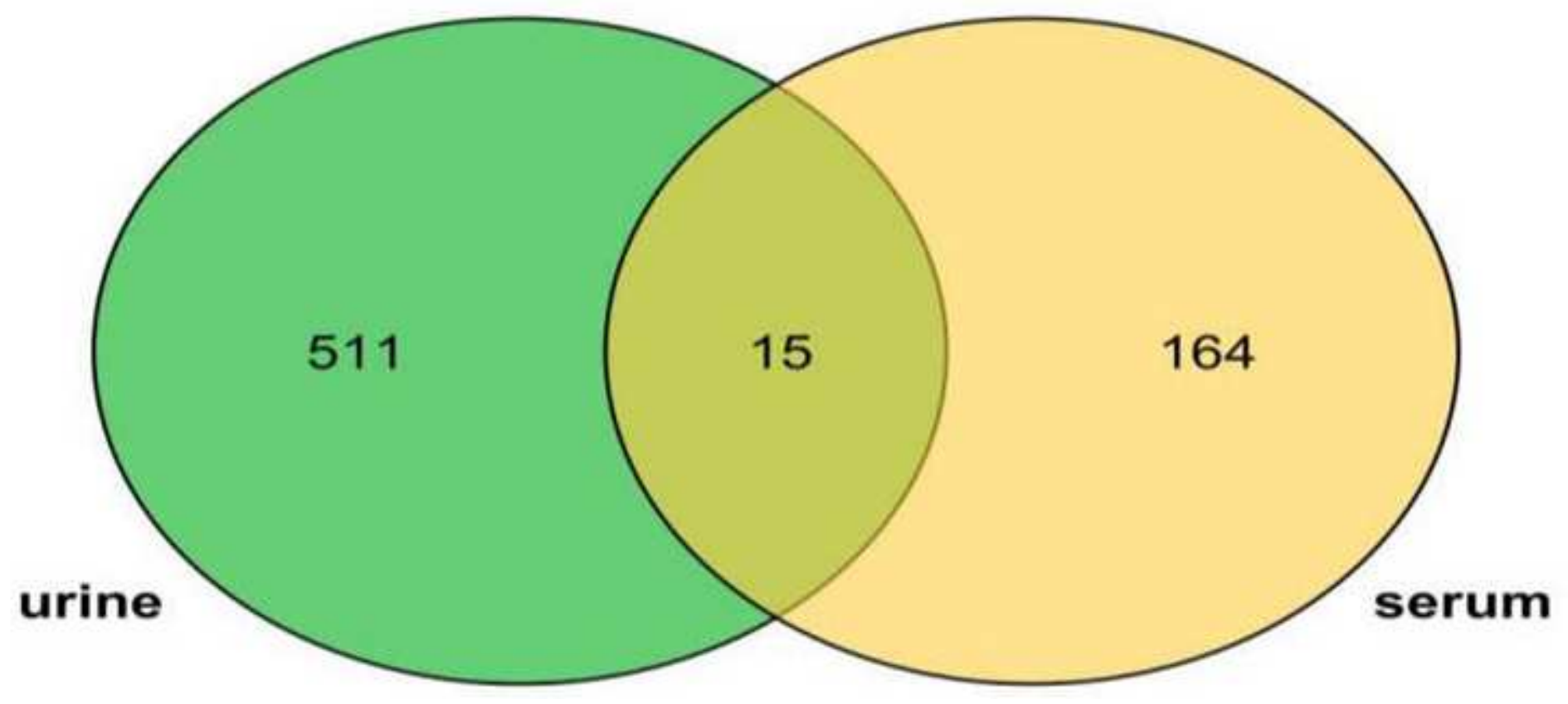

\section{Figure 6}

The bar graph of the correlation of spicific diseases and associated metabolites in serum (left) and urine (right). X-axis represents the number of assocated metabolites and y-axis represents the spicific diseases profiled by QEA. 


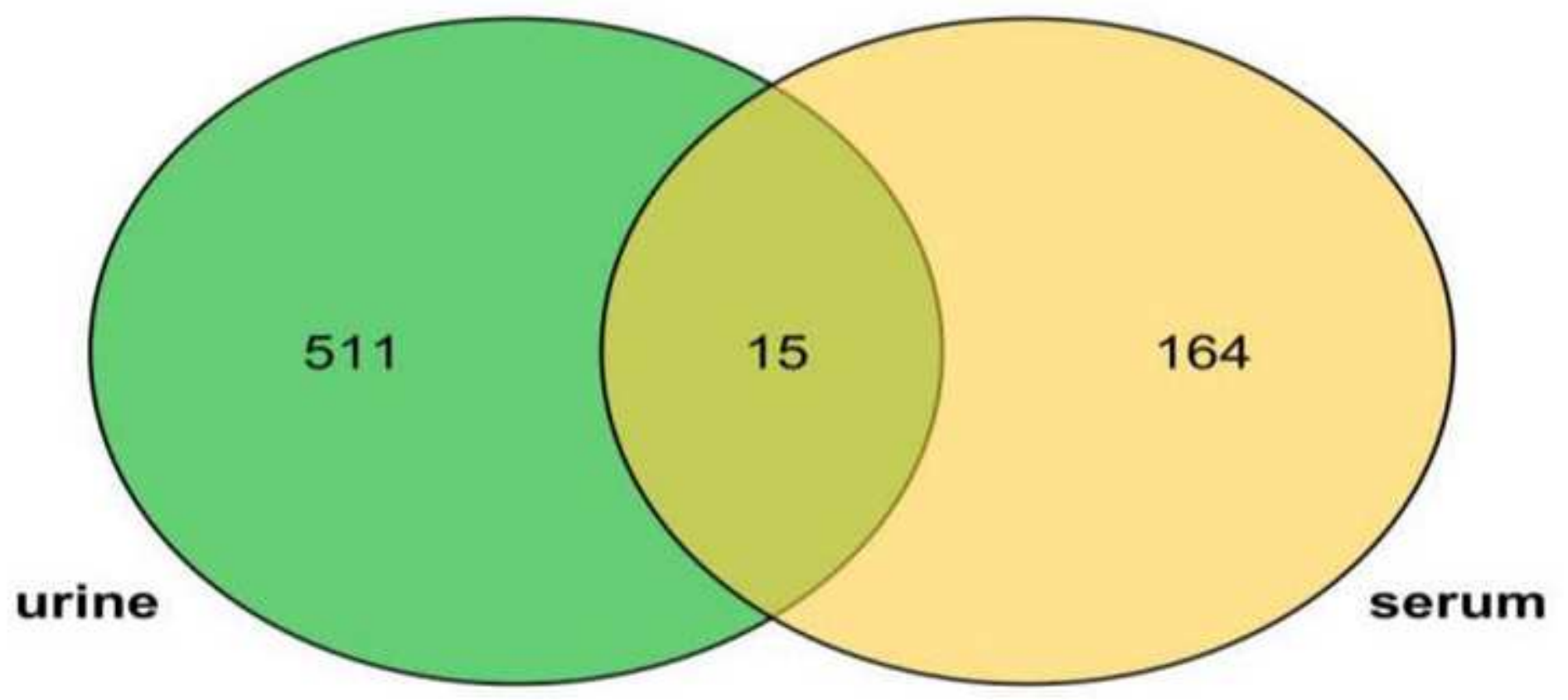

\section{Figure 6}

The bar graph of the correlation of spicific diseases and associated metabolites in serum (left) and urine (right). X-axis represents the number of assocated metabolites and $y$-axis represents the spicific diseases profiled by QEA.

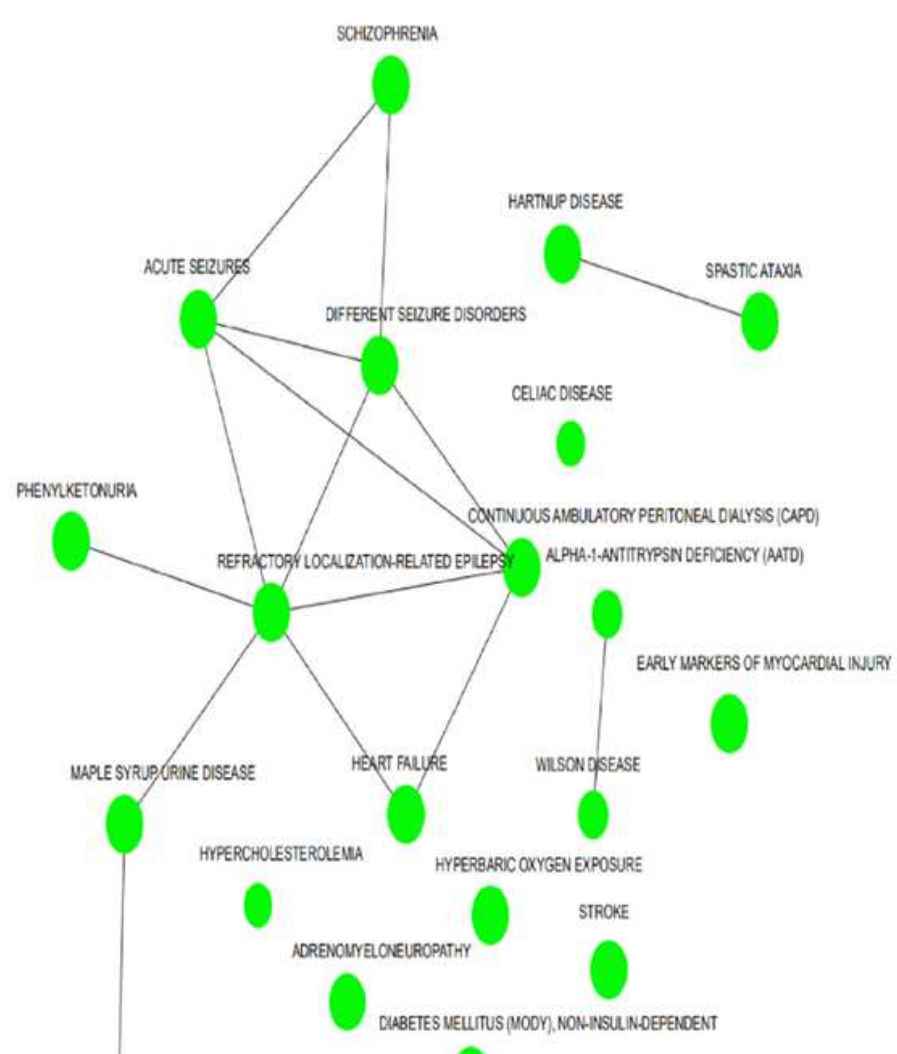

PYRUNAIE DEHYDROGEULSE DEFICENCY (E3)
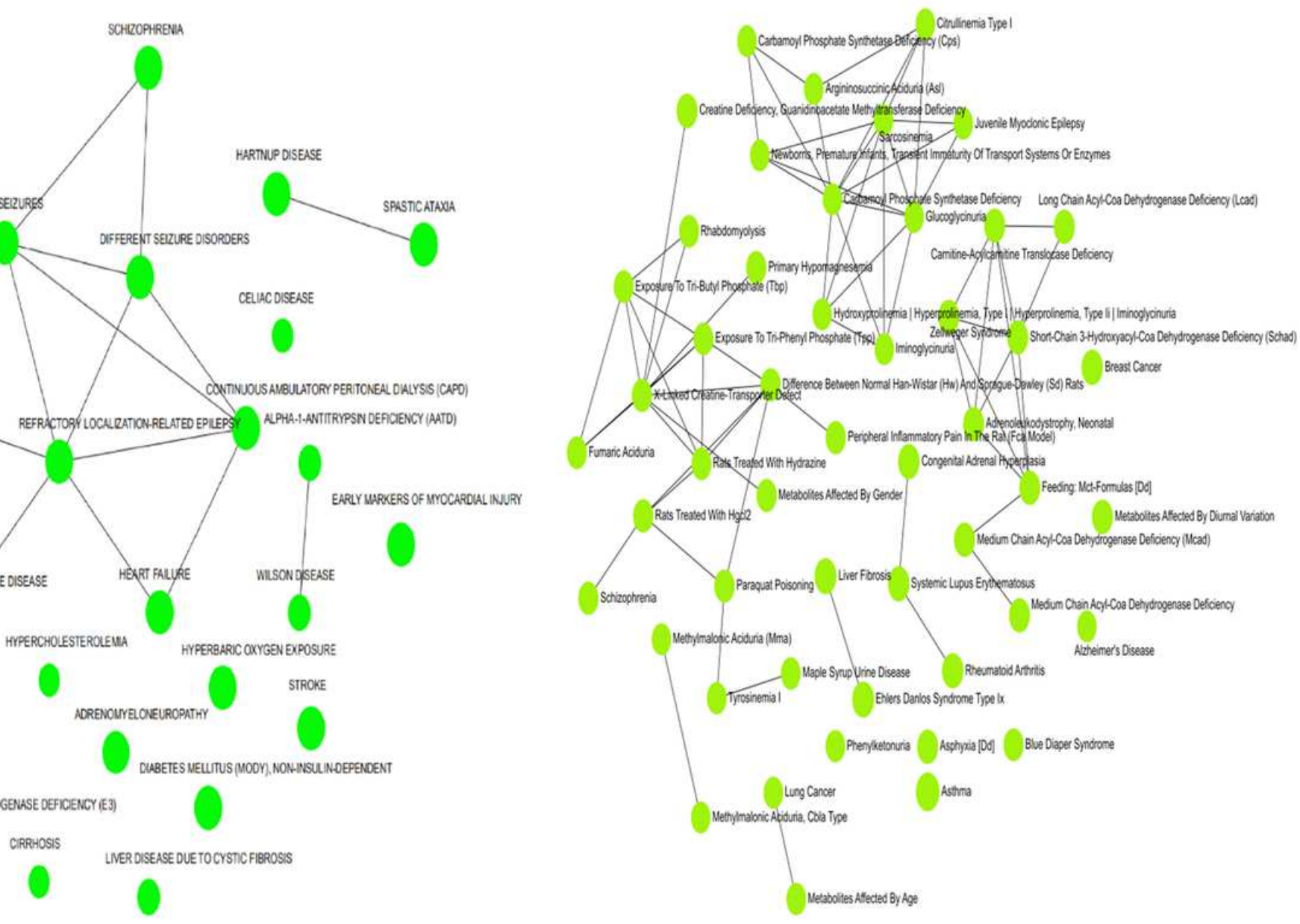
Figure 7

The network of the correlation of associatd diseases in serum (left) and urine (right).
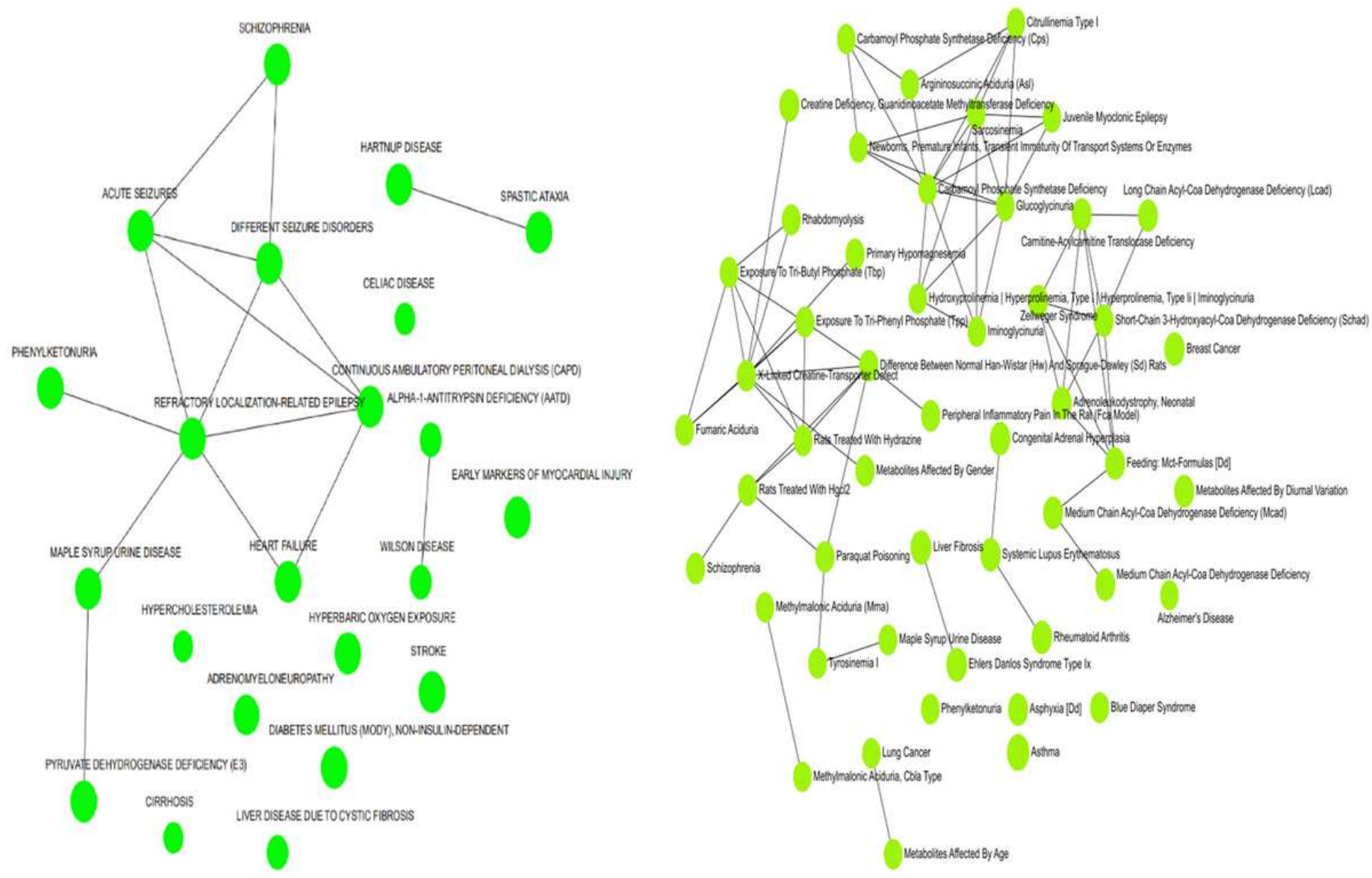

\section{Figure 7}

The network of the correlation of associatd diseases in serum (left) and urine (right). 

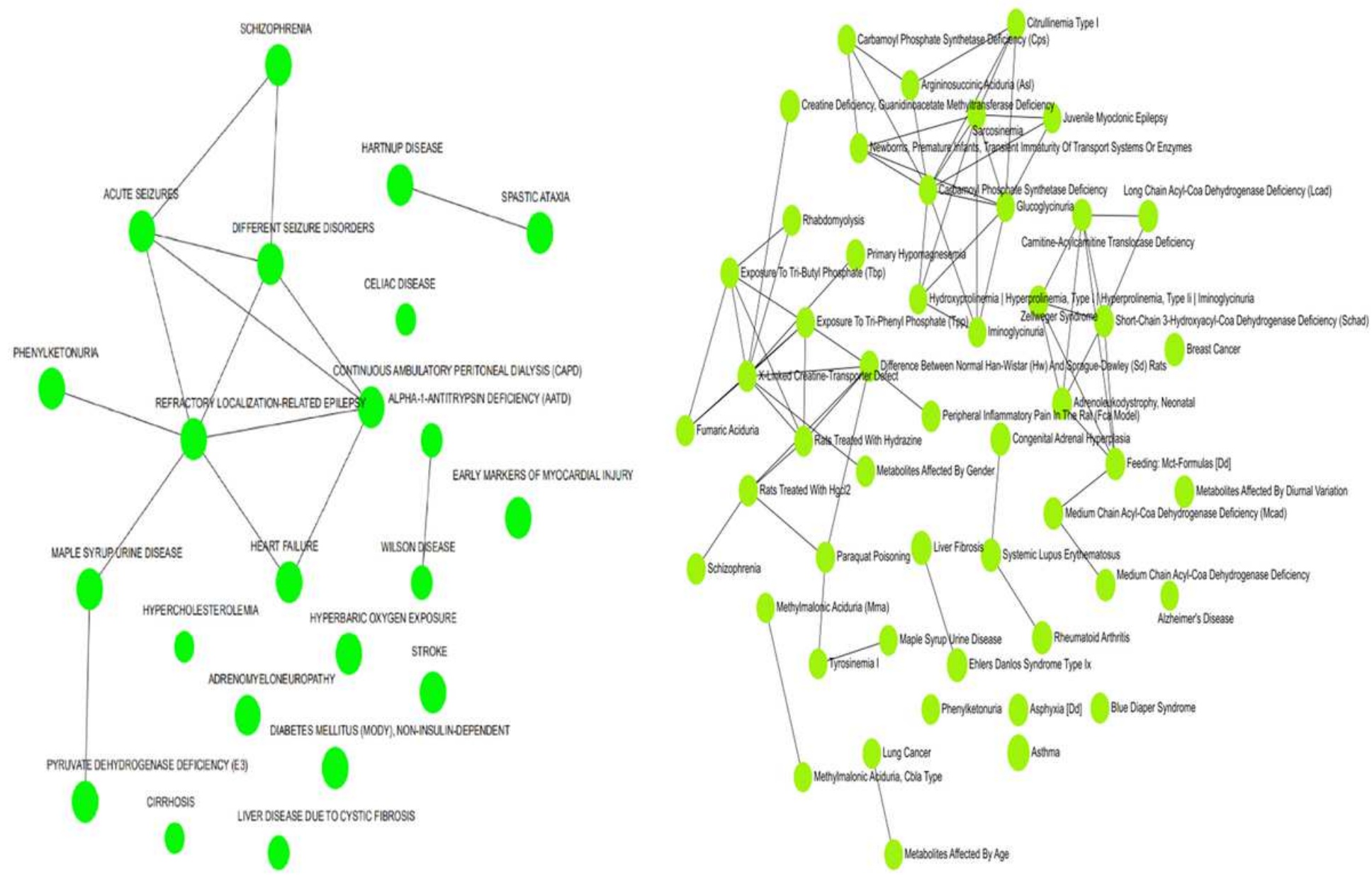

Figure 7

The network of the correlation of associatd diseases in serum (left) and urine (right).

\section{Supplementary Files}

This is a list of supplementary files associated with this preprint. Click to download.

- Tables1andTables2.doc

- Tables1andTables2.doc

- TableS1andTableS2.doc

- TableS3Differentialmetabolitesinserumandurine.xls

- TableS3Differentialmetabolitesinserumandurine.xls

- TableS3Differentialmetabolitesinserumandurine.xls

- TableS4Diseasesandmetabolitesinserumandurine.csv

- TableS4Diseasesandmetabolitesinserumandurine.csv

- TableS4Diseasesandmetabolitesinserumandurine.csv 